\title{
The Molecular Outflow in NGC 253 at a Resolution of Two Parsecs
}

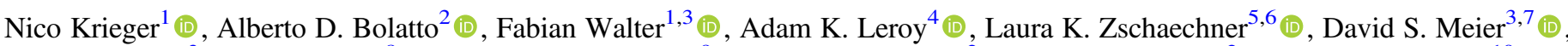 \\ Jürgen $\mathrm{Ott}^{3}$ (D), Axel Weiss ${ }^{8}$ (D), Elisabeth A. C. Mills ${ }^{9}$ (D), Rebecca C. Levy ${ }^{2}$ (D), Sylvain Veilleux ${ }^{2}$ (D), and Mark Gorski ${ }^{10}$ \\ ${ }^{1}$ Max-Planck-Institut für Astronomie, Königstuhl 17, D-69120 Heidelberg, Germany; krieger@mpia.de \\ ${ }^{2}$ Department of Astronomy, University of Maryland, College Park, MD 20742, USA \\ ${ }^{3}$ National Radio Astronomy Observatory, P.O. Box O, 1003 Lopezville Road, Socorro, NM 87801, USA \\ ${ }^{4}$ Department of Astronomy, The Ohio State University, 4055 McPherson Laboratory, 140 West 18th Avenue, Columbus, OH 43210, USA \\ ${ }^{5}$ Finnish Center for Astronomy with ESO, FI-20014 Turun yliopisto, Finland \\ ${ }^{6}$ University of Helsinki, P.O. Box 64, Gustaf Hällströmin katu 2a, FI-00014 Helsingin yliopisto, Finland \\ ${ }^{7}$ New Mexico Institute of Mining and Technology, 801 Leroy Place, Socorro, NM 87801, USA \\ ${ }^{8}$ Max-Planck-Institut für Radioastronomie, Auf dem Hügel 69, D-53121 Bonn, Germany \\ ${ }^{9}$ Physics Department, Brandeis University, 415 South Street, Waltham, MA 02453, USA \\ ${ }^{10}$ Department of Physics and Astronomy, University of Western Ontario, London, Ontario N6A 3K7, Canada \\ Received 2019 March 15; revised 2019 June 26; accepted 2019 June 26; published 2019 August 12
}

\begin{abstract}
We present 0." 15 ( $\sim 2.5 \mathrm{pc})$ resolution ALMA CO(3-2) observations of the starbursting center in NGC 253. Together with archival ALMA $\mathrm{CO}(1-0)$ and $\mathrm{CO}(2-1)$ data, we decompose the emission into disk and nondisk components. We find $\sim 7 \%-16 \%$ of the $\mathrm{CO}$ luminosity to be associated with the nondisk component $\left(1.2-4.2 \times 10^{7} \mathrm{~K} \mathrm{~km} \mathrm{~s}^{-1} \mathrm{pc}^{2}\right)$. The total molecular gas mass in the center of NGC 253 is $\sim 3.6 \times 10^{8} M_{\odot}$ with $\sim 0.5 \times 10^{8} M_{\odot}(\sim 15 \%)$ in the nondisk component. These measurements are consistent across independent mass estimates through three $\mathrm{CO}$ transitions. The high-resolution $\mathrm{CO}(3-2)$ observations allow us to identify the molecular outflow within the nondisk gas. Using a starburst conversion factor, we estimate the deprojected molecular mass outflow rate, kinetic energy, and momentum in the starburst of NGC 253. The deprojected molecular mass outflow rate is in the range of $\sim 14-39 M_{\odot} \mathrm{yr}^{-1}$ with an uncertainty of 0.4 dex. The large spread arises due to different interpretations of the kinematics of the observed gas while the errors are due to unknown geometry. The majority of this outflow rate is contributed by distinct outflows perpendicular to the disk, with a significant contribution by diffuse molecular gas. This results in a mass-loading factor $\eta=\dot{M}_{\text {out }} / \dot{M}_{\mathrm{SFR}}$ in the range $\eta \sim 8-20$ for gas ejected out to $\sim 300 \mathrm{pc}$. We find the kinetic energy of the outflow to be $\sim 2.5-4.5 \times 10^{54} \mathrm{erg}$ and a typical error of $\sim 0.8 \mathrm{dex}$, which is $\sim 0.1 \%$ of the total or $\sim 8 \%$ of the kinetic energy supplied by the starburst. The outflow momentum is $4.8-8.7 \times 10^{8} M_{\odot} \mathrm{km} \mathrm{s}^{-1}(\sim 0.5 \mathrm{dex}$ error $)$ or $\sim 2.5 \%-4 \%$ of the kinetic momentum released into the ISM by the feedback. The unknown outflow geometry and launching sites are the primary sources of uncertainty in this study.
\end{abstract}

Key words: galaxies: individual (NGC 253) - galaxies: ISM - galaxies: starburst - ISM: jets and outflows - ISM: kinematics and dynamics

\section{Introduction}

Outflows driven by star formation are thought to be a crucial driver of galaxy evolution. Strong stellar feedback caused by high star formation rate (SFR) densities can launch outflows of ionized, neutral, and molecular gas that can potentially escape the main body of a galaxy. Consequently, such outflowing gas removes the potential fuel for future star formation. Therefore, outflows can suppress and quench star formation, as also demonstrated by theoretical predictions and simulations (e.g., Dekel \& Silk 1986; Krumholz et al. 2017; Ma et al. 2018). Depending on the velocity of the outflow and a galaxy's escape velocity, outflowing gas can be reaccreted at later cosmic times (the so-called "galactic fountain") or leave the system altogether. This process thus has the potential to enrich the galactic disk and circumgalactic medium with heavy metals (e.g., Oppenheimer \& Davé 2006; Oppenheimer et al. 2010; Hopkins et al. 2012; Christensen et al. 2018).

Galactic outflows are a multiphase phenomenon and are observed across the electromagnetic spectrum from X-ray (e.g., Strickland \& Heckman 2007), UV (e.g., Hoopes et al. 2005), optical like $\mathrm{H} \alpha$ (e.g., Westmoquette et al. 2009), to IR (e.g., Veilleux et al. 2009), cold dust (e.g., Roussel et al. 2010), PAH emission (e.g., Engelbracht et al. 2006), and submillimeter to radio including H I (e.g., Bolatto et al. 2013; Leroy et al. 2015b; Lucero et al. 2015). Typically, large-scale outflow features at high relative velocity (hundreds to thousands of $\mathrm{km} \mathrm{s}^{-1}$ ) are observed in the ionized and neutral gas, whereas molecular outflows often appear as smaller, more compact features (Strickland et al. 2002; Westmoquette et al. 2011). The latter are nonetheless important as they dominate the mass budget (Leroy et al. 2015b). In some galaxies, the gas phases seem to be stratified with an inner ionized outflow cone, a surrounding neutral shell, and molecular gas situated along the outer edge (e.g., Meier et al. 2015) Typically, the outflows originate from an extended region, so the apparent outflow cone has its tip cut off.

Molecular outflows are thus closely intertwined with feedback processes and star formation. The high-resolution structure and kinematic properties of (molecular) outflows have not been studied in great detail yet, primarily due to the lack of high-resolution and sensitivity observations. Starburst galaxies are the obvious target to study star-formation-driven outflows due to the high SFR in these systems. Consequently, molecular outflows have been studied over the past years in a few nearby starbursts: M82 (Walter et al. 2002; Leroy et al. 2015b), NGC 253 (Bolatto et al. 2013; Walter et al. 2017; Zschaechner et al. 2018), NGC 1808 (Salak et al. 2018), and ESO320-G030 (Pereira-Santaella et al. 2016). 
Table 1

Details of the Data Sets Used in This Analysis

\begin{tabular}{|c|c|c|c|}
\hline & $\mathrm{CO}(1-0)$ & $\mathrm{CO}(2-1)$ & $\mathrm{CO}(3-2)$ \\
\hline ALMA ID & 2011.1.00172.S & 2012.1.00108.S & 2015.1.00274.S \\
\hline \multirow[t]{2}{*}{ Spatial Resolution } & $1 " .85 \times 1 ! .32$ & $1 "$. $70 \times 1 "$ × 02 & $0 "$ " $17 \times 0$ " 13 \\
\hline & $31.4 \mathrm{pc} \times 22.4 \mathrm{pc}$ & $28.8 \mathrm{pc} \times 17.3 \mathrm{pc}$ & $2.9 \mathrm{pc} \times 2.2 \mathrm{pc}$ \\
\hline Spectral Resolution & $5.0 \mathrm{~km} \mathrm{~s}^{-1}$ & $5.0 \mathrm{~km} \mathrm{~s}^{-1}$ & $2.5 \mathrm{~km} \mathrm{~s}^{-1}$ \\
\hline \multirow[t]{2}{*}{ rms Noise Per Channel } & $1.99 \mathrm{mJy}^{\text {beam }^{-1}}$ & $2.19 \mathrm{mJy}^{\text {beam }^{-1}}$ & $0.81 \mathrm{mJy}^{\text {beam }^{-1}}$ \\
\hline & $75 \mathrm{mK}$ & $29 \mathrm{mK}$ & $0.37 \mathrm{~K}$ \\
\hline
\end{tabular}

NGC 253 is one of the nearest starburst systems at a distance of $3.5 \mathrm{Mpc}$ (Rekola et al. 2005). It is considered one of the prototypical starburst galaxies with an SFR surface density of $\Sigma_{\mathrm{SFR}} \sim 10^{2} M_{\odot} \mathrm{yr}^{-1} \mathrm{kpc}^{-2}$ in the nuclear region and a molecular depletion time that is $\tau_{\text {dep }}^{\text {mol }} \sim 5-25$ times lower than what is found in local disks (Leroy et al. 2015a). A galactic wind emerges from the central $\sim 200$ pc of NGC 253, which has been characterized in $\mathrm{H} \alpha, \mathrm{X}$-ray, as well as neutral and molecular gas emission (e.g., Turner 1985; Heckman et al. 2000; Strickland et al. 2000, 2002; Sharp \& Bland-Hawthorn 2010; Sturm et al. 2011; Westmoquette et al. 2011; Bolatto et al. 2013; Walter et al. 2017). Due to the close proximity, starburst and galactic winds can be studied in detail and individual structures can be resolved.

Studies of the molecular gas phase in NGC 253 showed that its central starburst is fueled by gas accretion along the bar (Paglione et al. 2004). The molecular ISM in the nuclear region is structured in several clumps that show high temperatures of 50 K (Paglione et al. 2004; Sakamoto et al. 2011; Mangum et al. 2019). From earlier low-resolution observations ( $>20 \mathrm{pc}$, e.g., Sakamoto et al. 2006, 2011) to recent observations at high resolution $(8 \mathrm{pc} \times 5 \mathrm{pc}$ in Ando et al. 2017 and $2 \mathrm{pc}$ in Leroy et al. 2018), the number of molecular clumps associated with the starburst has increased from $\sim 5$ to 14 . These studies find the clumps to be massive $\left(4-10 \times 10^{4} M_{\odot}\right)$, compact $(<10 \mathrm{pc})$, chemically rich (up to $>19$ molecules detected in the $0.8 \mathrm{~mm}$ band), and hot (up to $90 \mathrm{~K}$ ). Each clump likely hosts an embedded massive star cluster (Leroy et al. 2018). Further structures in the molecular gas are shells and bubbles blown up by feedback from the intense star formation process. Sakamoto et al. (2006) found two $100 \mathrm{pc}$ diameter superbubbles. Bolatto et al. (2013) reported molecular streamers ${ }^{11}$ originating from these shells with a lower limit on the outflow rate of 3-9 $M_{\odot} \mathrm{yr}^{-1}$, about three times the SFR. This estimate was revisited by Zschaechner et al. (2018), based on observations that show that the $\mathrm{CO}$ emission associated with the most prominent streamer is optically thick, increasing it to $25-50 M_{\odot} \mathrm{yr}^{-1}$.

As suggested by these studies, the outflow rate in NGC 253 is factors of a few to potentially $>10$ larger than the SFR. Hence, the impact of the outflows on the amount of material lost from the molecular gas reservoir, and thus the lifetime of the starburst, is significant. The availability of new data makes it interesting to revisit the determination of the mass outflow rate in NGC 253, while also removing some limitations of previous determinations. Bolatto et al. (2013) estimated the outflow rate from a few massive molecular streamers, but did not include potential diffuse outflowing gas. Also, resolution

\footnotetext{
11 The term streamer here denotes structures with a high aspect ratio that are typically oriented roughly perpendicular to the disk and often show a velocity gradient.
}

plays an important role in the ability to disentangle outflows from material in the starbursting disk. New ALMA band 7 observations provide excellent spatial resolution and reasonable surface brightness sensitivity. This information enables increasingly accurate determination of the total mass outflow rate and its impact on the starburst.

In this work, we present ALMA $\mathrm{CO}(3-2)$ observations carried out in cycles 3 and 4 that target the molecular gas in the central $\sim 750 \mathrm{pc}$ of NGC 253 . Together with ancillary band 3 and 6 data from our previous works (Bolatto et al. 2013; Leroy et al. 2015a; Meier et al. 2015; Zschaechner et al. 2018), we have an inventory of three CO lines to study the molecular gas in the starbursting disk and a kinematically different component that includes the outflow. By decomposing the detected emission, we aim to measure the total molecular gas outflow rate in NGC 253 and improve upon previous less systematic results.

Throughout this paper, we adopt a distance of $3.5 \mathrm{Mpc}$ to NGC 253 (Rekola et al. 2005) at which $1^{\prime \prime}$ corresponds to $17 \mathrm{pc}$. We also define the "center" of the nuclear region of NGC 253 to be the kinematic center at $\alpha, \delta=00^{\mathrm{h}} 47^{\mathrm{m}} 33.134,-25^{\circ} 17^{\mathrm{m}} 19.68$, as identified in Müller-Sánchez et al. (2010). The paper is structured as follows: in Section 2, we describe observational setup and data reduction, and show the results in the form of channel maps, moment maps, and position-velocity $(\mathrm{pV})$ diagrams. Our approach to separating gas in the star-forming disk from potentially outflowing gas is laid out in Section 3. Section 4 discusses the derived quantities such as the CO luminosities, molecular gas masses, outflow rates, kinetic energies, and momenta. Our conclusions are summarized in Section 5.

\section{Data Reduction and Imaging}

\subsection{Data Reduction}

The data presented in this paper are based on observations in ALMA cycles 2, 3, and 4 in bands 3,6 , and 7 that cover the redshifted emission in NGC 253 of $\mathrm{CO}(1-0), \mathrm{CO}(2-1)$, and $\mathrm{CO}(3-2)$, as well as other molecular lines. For the data reduction and imaging of band 3 and 6 data, see Bolatto et al. (2013), Leroy et al. (2015a), Meier et al. (2015), and Zschaechner et al. (2018). Table 1 gives an overview of the data sets used in this analysis.

For the band 7 observations, we tuned the lower sideband to $342.0-345.8 \mathrm{GHz}$ and the upper sideband to $353.9-357.7 \mathrm{GHz}$ (total bandwidth $7.6 \mathrm{GHz}$ ) with a $976.6 \mathrm{kHz}$ channel width (corresponding to $0.8 \mathrm{~km} \mathrm{~s}^{-1}$ ). We targeted the central $\sim 750 \mathrm{pc}$ of NGC 253 in a linear four-pointing mosaic with two configurations of the $12 \mathrm{~m}$ array $(12 \mathrm{~m}$ compact and $12 \mathrm{~m}$ extended, half-power beam width $\sim 30^{\prime \prime}$ ) and a five-pointing mosaic of the $7 \mathrm{~m}$ array (ACA, half-power beam width $\sim 50^{\prime \prime}$ ). Additional single-dish observations with the total power array 
(TP) recovers emission on large spatial scales. The baseline ranges covered by this setup are $8.9-49.0 \mathrm{~m}, 15.1-783.5 \mathrm{~m}$, and $15.1-1813.1 \mathrm{~m}$ for the ACA and the two $12 \mathrm{~m}$ setups, respectively.

The observations were carried out primarily in the first half of 2016 (TP: 2015 December 7 to 2016 August 2; ACA: 2015 December 7 to 2016 November 23; $12 \mathrm{~m}$ compact configuration: 2016 April 16, 2016 April 23, 2016 June 17, 2016 June 27; $12 \mathrm{~m}$ extended configuration: 2016 August 30, 2016 September 3). The total on-source observation time is $48 \mathrm{hr}$ and 45 minutes split across $27 \mathrm{hr}$ and 23 minutes (TP), $14 \mathrm{hr}$ and 57minutes (ACA), $2 \mathrm{hr}$ and 37 minutes (12 m compact), and $3 \mathrm{hr}$ and 59 minutes ( $12 \mathrm{~m}$ extended). The calibrators were J0006-0623 (bandpass); J0038-2459 (complex gain); the asteroid Pallas (absolute flux density); and J0104-2416 and J0106-2718 (both WVR). Visibilities of the $12 \mathrm{~m}$ data are calibrated using the ALMA cycle 3 pipeline in CASA 4.6.0 and the delivered calibration script. The other data sets are calibrated in CASA 4.7.2 and the cycle 4 pipeline.

In order to image the spectral lines, we subtract the continuum in the $(U, V)$ plane using a first-order polynomial fitted to the channels that do not contain strong spectral lines. We reliably detect $>25$ lines in the range $342.0-345.0 \mathrm{GHz}$ and $353.9-357.7 \mathrm{GHz}$ aside from the four strong lines $\mathrm{CO}(3-2)$, HCN4-3), $\mathrm{HCO}^{+}(4-3)$, and $\mathrm{CS}(7-6)$. Most of these lines are weak and only detected in small spatial regions so they do not affect the overall continuum fit and subtraction.

\subsection{Imaging}

Combined imaging of the interferometric data is done with the tclean task in CASA 5.4.0, which includes crucial bug fixes for ALMA mosaics. ${ }^{12}$ We regrid the visibilities during deconvolution to a spectral resolution of $2.5 \mathrm{~km} \mathrm{~s}^{-1}$. Applying a Briggs weighting scheme with robust parameter 0.5 results in a synthesized beam of 0 ! $17 \times 0$." 13 (pixel scale 0 ". 05 ). The images are cleaned to a level of $2.5 \times$ the rms noise in line-free channels of $2.5 \times 0.81 \mathrm{mJy}^{-1}$ beam $^{-1}(2.5 \times 0.37 \mathrm{~K})$ using a clean mask derived from a low-resolution image of the compact $12 \mathrm{~m}$ array $\mathrm{CO}(3-2)$ data only.

We correct the cleaned images for the mosaic sensitivity pattern (mosaic primary beam response pattern), combine them with the TP images using feather, and finally convert the units to brightness temperature.

For the final images, we do not consider the ACA data as they introduce large-scale noise fluctuations toward the edge of the mosaic, which we attribute to decreasing sensitivity of the $12 \mathrm{~m}$ data relative to the ACA data. These fluctuations obscure the regions where outflows were previously found. This work requires accurate integrated flux measurements and correct representation of the small-scale structure, which are defined by single-dish observations (TP) and long baselines (extended $12 \mathrm{~m}$ ), respectively. By checking the images without ACA data against the images including ACA data, we can confirm that neither the overall flux scale nor the small-scale structure is significantly altered.

Data products for $\mathrm{CO}(1-0)$ are shown in Bolatto et al. (2013), Meier et al. (2015), and Leroy et al. (2015a), and

\footnotetext{
12 For details, see NAASC memo 117 by the North American ALMA Science Center (NAASC) at http://library.nrao.edu/public/memos/naasc/NAASC_ 117.pdf.
}

Zschaechner et al. (2018) present the $\mathrm{CO}(2-1)$ data. Imaging results for $\mathrm{CO}(3-2)$ are presented in the following section.

In order to keep the amount of detail and contrast in the highresolution data, we do not match the spatial resolution to that of the data with the lowest resolution, but perform our analysis at the native resolution of each data set. All further steps work on the data cubes masked at $5.0 \sigma$ (see Table 1) and further masks where necessary. To generate the masks, we do not consider the nonuniform noise level caused by the mosaic sensitivity pattern but use the per-channel rms noise in the center of the field of view.

\section{3. $C O(3-2)$ Data Presentation}

In this section, we present the $\mathrm{CO}(3-2)$ data in different representations. Channel maps (Figure 1), moment maps (Figure 2), and a pV diagram (Figure 3) show the spatial and kinematic structures to be discussed and highlight the data quality.

Figure 1 shows channel maps of the image cube. To retain the intrinsic resolution, only every 16 th channel ( $40 \mathrm{~km} \mathrm{~s}^{-1}$ spacing) is shown here. Aside from the rotating disk of molecular gas, we clearly detect the prominent southwest (SW) streamer (Walter et al. 2017) in the range 180-250 $\mathrm{km} \mathrm{s}^{-1}$ (Figure 1, panels 220 and $260 \mathrm{~km} \mathrm{~s}^{-1}$ ). Additional gas streamers are apparent between $\sim 60$ and $\sim 350 \mathrm{~km} \mathrm{~s}^{-1}$ toward the north and south of the disk, as can be seen, for example, in the panels at 260 or $340 \mathrm{~km} \mathrm{~s}^{-1}$. Several notable molecular shells are present between 180 and $340 \mathrm{~km} \mathrm{~s}^{-1}$. Aside from the (super)shells at the eastern (left) and western (right) edges of the map that were previously identified by Sakamoto et al. (2006) and Bolatto et al. (2013), further smaller shell-like structures are located along the molecular disk.

We calculate image moments (Figure 2) with immoments in CASA for emission above $5 \sigma$ for the moment 0 (integrated intensity), moment 1 (intensity-weighted line-of-sight velocity), and moment 2 (intensity-weighted velocity dispersion) maps. Note that due to the complex line shapes, the moment 2 map does not directly correspond to velocity dispersion, which is only the case for Gaussian line profiles. The maps are further constrained to the region defined by the collapsed clean mask to limit them to emission that has been processed by the clean algorithm.

Figure 3 shows the kinematic structure of NGC 253 in a pV diagram along the major axis of the disk $\left(\mathrm{PA}=55^{\circ}\right)$ averaged over the full width of the field of view $\left(\sim 30^{\prime \prime}\right)$ centered on the kinematic center. The $\mathrm{pV}$ cut shows several high-velocity dispersion structures extending from a rotating disk, indicative of outflows.

\section{Separating Disk and Nondisk Emission}

\subsection{Separating Disk and Nondisk Emission in Position- Position-Velocity ( $p p V)$ Space}

Our goal is to account for all molecular wind, separating outflowing molecular gas from foreground or background disk emission. A clean separation in 2D position-position space cannot be easily accomplished, due to the inclination of $78^{\circ}$ of NGC 253. At this high inclination, outflows and disk emission are cospatial in projection. Kinematic information from line-ofsight velocities, however, makes it possible to disentangle the outflow. Note that this becomes increasingly difficult as the velocity vector aligns with the plane of the sky, resulting in line-of-sight velocities that are systemic. From $\mathrm{H} \alpha$ kinematic modeling, the NGC 253 outflow is approximately biconical 


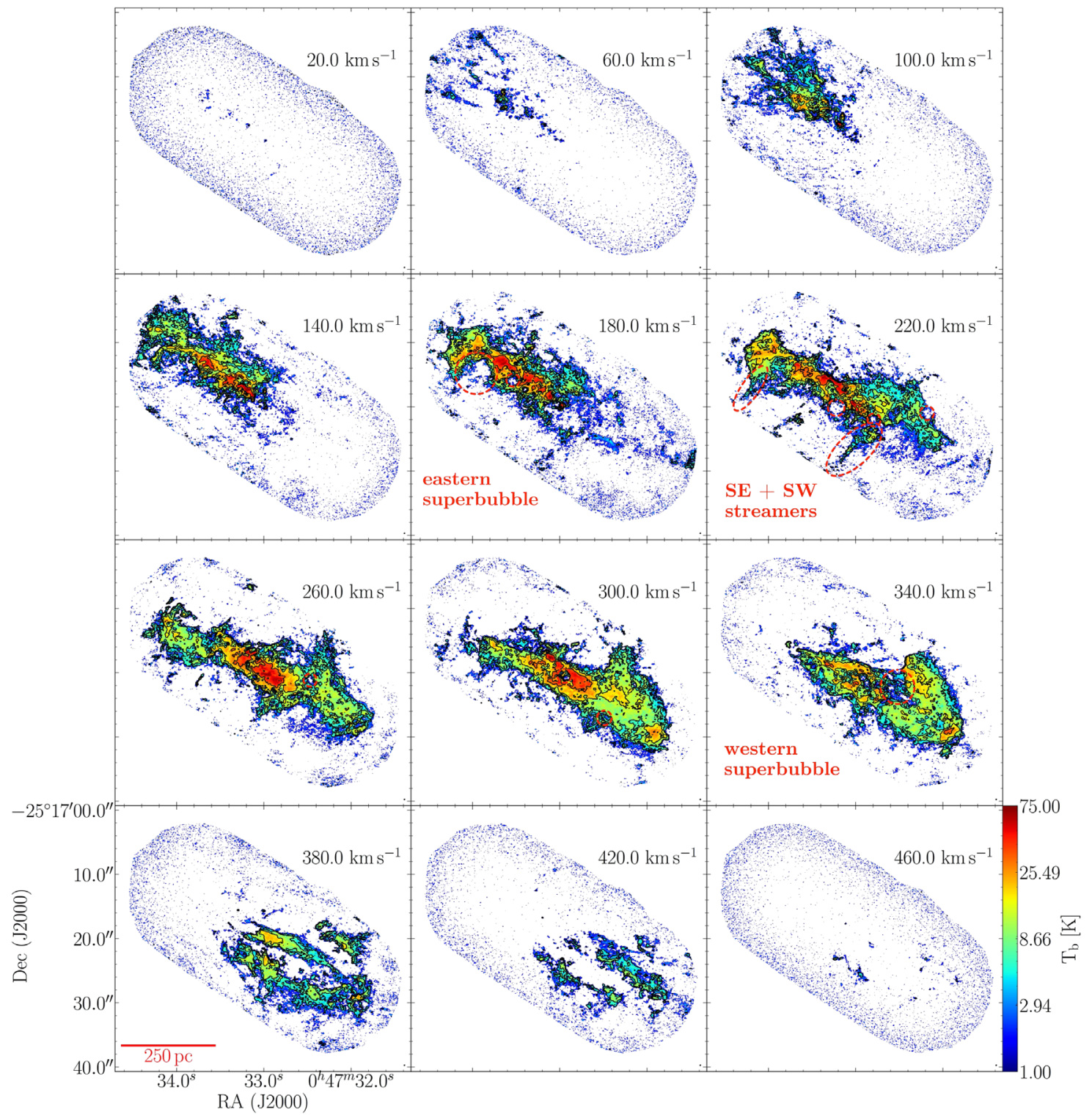

Figure 1. Channel maps of $\mathrm{CO}(3-2)$ in NGC 253. Every 16th channel of $2.5 \mathrm{~km} \mathrm{~s}^{-1}$ width is shown with the corresponding line-of-sight velocity $\left(v_{\mathrm{sys}}=250 \mathrm{~km} \mathrm{~s}{ }^{-1}\right)$ given in the upper-right corner of each panel. The synthesized beam of $0.17 \times 0.13$ is plotted in the lower-right corner; it is hardly noticeable due to its small size. Contours are plotted at $10 \sigma, 20 \sigma, 40 \sigma$, and $80 \sigma$ with an rms noise of $\sigma=0.37 \mathrm{~K}$. Large structures are marked by dashed contours in those panels that show them most clearly. Further new shells are indicated by dashed circles.

with an axis normal to the disk and an opening angle of $\sim 60^{\circ}$ (Westmoquette et al. 2011), and thus the range of possible projection angles is large (see Meier et al. 2015 for a sketch). Note that because the cone-opening angle is larger than the angle between the axis of the cone and the plane of the sky, gas in the approaching and receding cones can have both blue- and redshifted velocities with respect to systemic.

The launching of molecular gas occurs within the disk through star formation feedback, thus the outflows originate from the same location in $\mathrm{ppV}$ space as disk molecular clouds. Outflows will therefore blend into the disk near their launching sites, which makes disentanglement increasingly difficult closer to the starburst region.

The complexity of systematically separating the emission corresponding to the disk and the outflow in $\mathrm{ppV}$ space is challenging. Algorithmically, this separation is simpler in lower dimensional space, obtained by slicing the data cube into a collection of $2 \mathrm{D} \mathrm{pV}$ diagrams. In what follows, we identify kinematic components in these diagrams, which we then project back to $3 \mathrm{D} \mathrm{ppV}$ space. In order to avoid introducing biases, we model the large-scale disk velocity field and use this model as the basis of the kinematic separation.

\subsection{Definition of Components}

Images of the center of NGC 253 on large scales show an elongated gas structure (Figure 2 top) with a regular velocity field (Figure 2 middle) that roughly matches a rotating disk disturbed by streaming motions from a bar (e.g., Paglione et al. 2004). The elongated gas structure is consistent with a highly inclined disk of molecular gas, or possibly a ring-like structure as observed in other galaxies (for example, NGC 1512, NGC 1808; Salak et al. 2016; Ma et al. 2018). Similar structures break up at higher spatial resolution into two embracing spiral arms or complex nonclosed orbits in the 


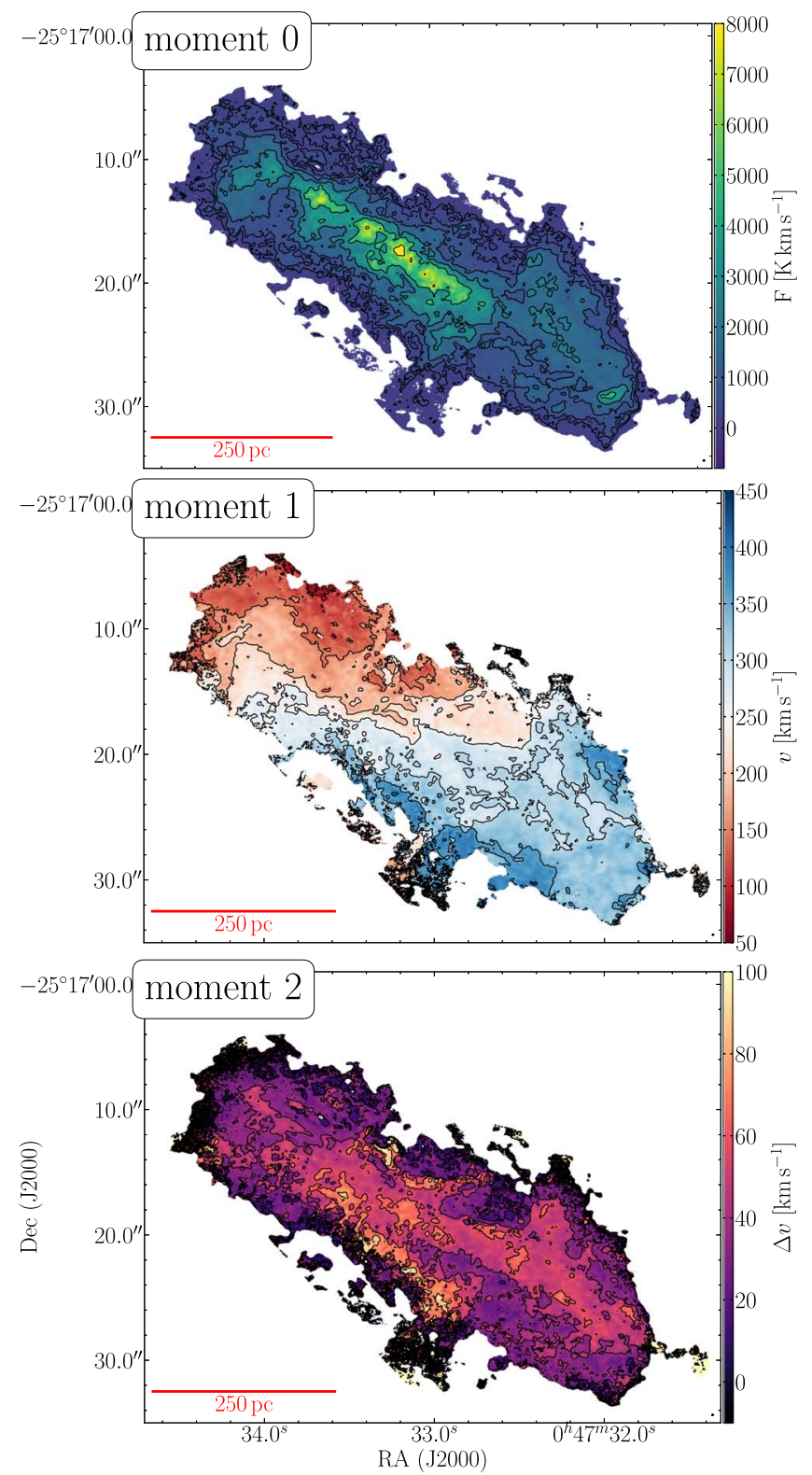

Figure 2. $\mathrm{CO}(3-2)$ moment maps of NGC 253. Top: integrated intensity map (moment 0); contours are shown from 250 to $8000 \mathrm{~K} \mathrm{~km} \mathrm{~s}^{-1}$ in factors of 2 . Middle: velocity field (moment 1); contours are shown from 100 to $400 \mathrm{~km} \mathrm{~s}^{-1}$ in steps of $50 \mathrm{~km} \mathrm{~s}^{-1}$. Bottom: moment 2 (corresponding to the velocity dispersion if the line profile were Gaussian); contours are shown from 0 to $100 \mathrm{~km} \mathrm{~s}^{-1}$ in steps of $20 \mathrm{~km} \mathrm{~s}^{-1}$. The color scale is chosen to saturate a few regions with dispersions $>100 \mathrm{~km} \mathrm{~s}^{-1}$. All maps are generated from the data cube masked at $5 \sigma$ threshold per channel and confined to the collapsed clean mask to include only emission that has been processed by the clean algorithm.

Milky Way center (Krumholz \& Kruijssen 2015; Henshaw et al. 2016; Sormani et al. 2018).

Superimposed on this large-scale structure are smaller features that are not part of the large-scale pattern of rotation and streaming motions. Some of them have high aspect ratios in channel maps and line-of-sight velocity gradients, both typical for outflows. Local deviations from the large-scale velocity field can also be due to infalling gas, or clumps of gas that do not follow the global pattern, due perhaps to cloudcloud collision.

Henceforth, we will refer to the bulk of the molecular gas that moves according to the large-scale velocity field in the

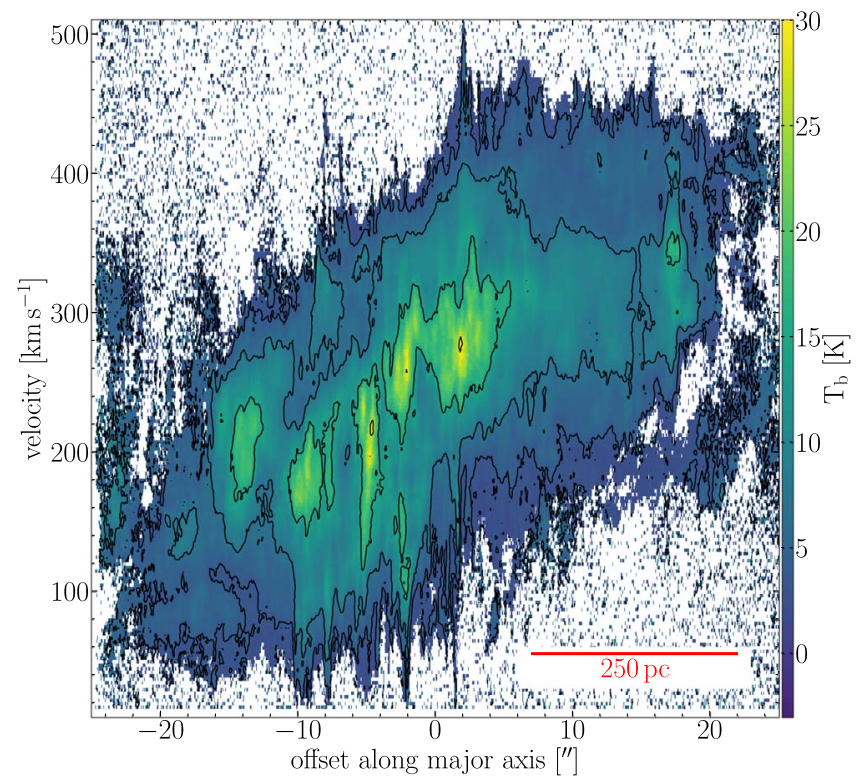

Figure 3. $\mathrm{CO}(3-2)$ position-velocity diagram of NGC 253 along the major axis centered on the kinematic center averaged over the full width of the field of view $\left(\sim 30^{\prime \prime}\right)$. Pixels below $3 \sigma$ are masked and contours are drawn at $10 \sigma, 20 \sigma$, $40 \sigma, 80 \sigma$ with an rms noise of $\sigma=0.37 \mathrm{~K}$. Note the vertical spikes indicating high-velocity dispersion due to outflowing gas.

central regions of NGC 253 as the disk. We assume that this large-scale velocity field consists of rotation and streaming motions. The term nondisk refers to any gas that is not following the $\mathrm{ppV}$ structure of the disk. By this definition, nondisk gas encompasses material from features that may be attributed to a variety of physical processes, including outflow and infall.

Structures of outflowing gas are frequently referred to by names that describe their kinematic or spatial appearance, such as "streamer." We will use the term outflow to denote localized structures with morphology and kinematics consistent with gas moving away from the disk, as inferred from their location in $\mathrm{ppV}$ space. Typical signatures are a velocity that is inconsistent with rotation in the plane of the disk and a high aspect ratio oriented roughly perpendicular to the disk major axis. Note that similar kinematic and structural properties can arise in infalling gas clouds. We will assume that all molecular gas with these characteristics is outflowing, which is likely the case for the majority of the material in NGC 253.

\subsection{Position-Velocity Slicing}

Kinematic analyses typically depend on high signal-to-noise ratios $(\mathrm{S} / \mathrm{N})$ because faint features can easily drown in noisy spectra. As a trade-off between necessarily high $\mathrm{S} / \mathrm{N}$ and also trying to include as much faint emission as possible, we conduct the following analysis on data cubes masked at the $5 \sigma$ level (see Table 1).

We split the ppV cubes into $\mathrm{pV}$ slices along the major axis of NGC 253 as shown in Figure 4. The slices assume the kinematic center is $\alpha, \delta=00^{\mathrm{h}} 47^{\mathrm{m}} 33^{\mathrm{s}} .134,-25^{\circ} 17^{\mathrm{m}} 19^{\mathrm{s}} .68$ (Müller-Sánchez et al. 2010), and are oriented along the major axis of the projected $\mathrm{CO}$ emission with $\mathrm{PA}=55^{\circ}$. The area sliced is chosen to cover the region for which we have overlapping $\mathrm{CO}(1-0),(2-1)$, and (3-2), and also cover the full length of the SW streamer outflow feature (17!"5; Walter et al. 2017). 

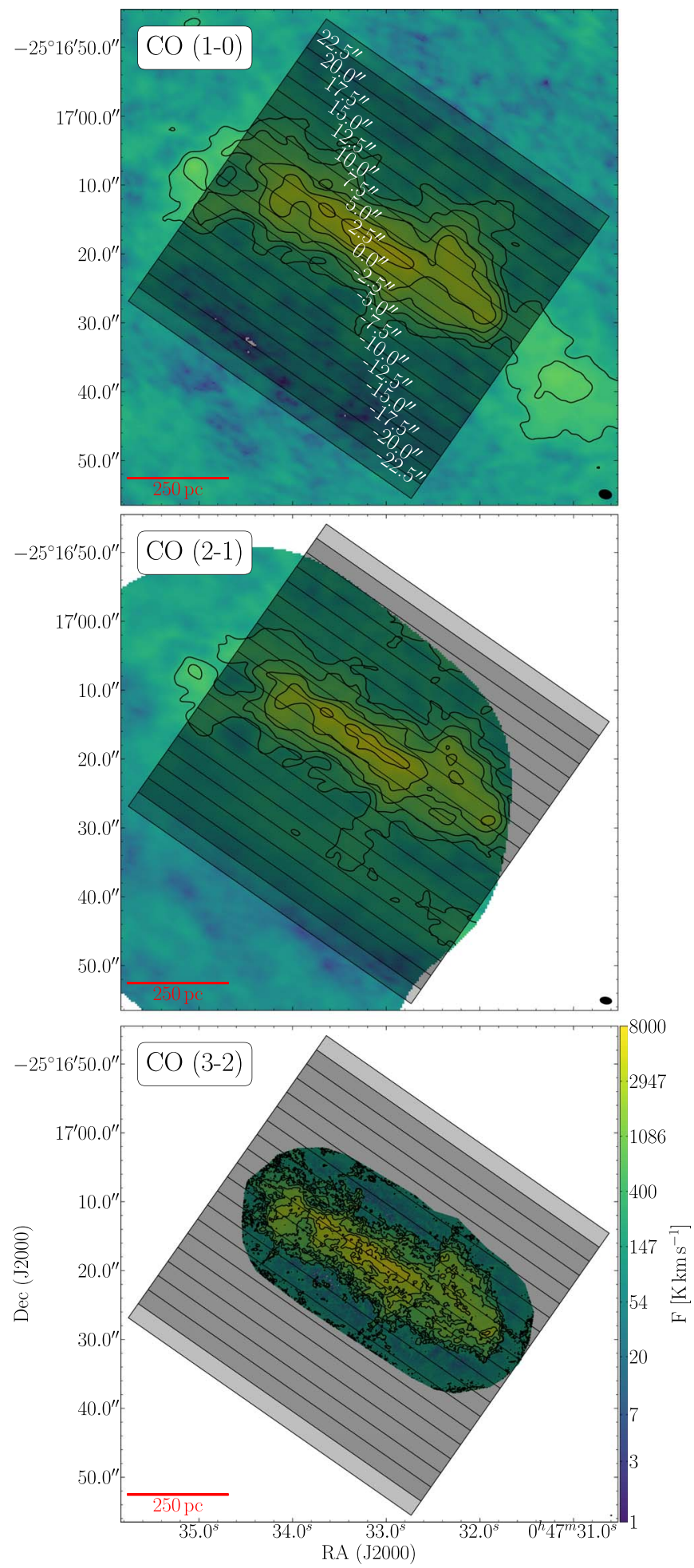

Figure 4. Size and orientation of the position-velocity slices overlaid on the integrated intensity image of $\mathrm{CO}(1-0)$ (top), $\mathrm{CO}(2-1)$ (middle), and $\mathrm{CO}(3-2)$ (bottom). Each slice is 5 !" 0 wide and overlaps adjacent slices by 2 ". 5 .

These requirements are fulfilled by slices of $50^{\prime \prime}(850 \mathrm{pc})$ length (major axis) and covering $50^{\prime \prime}(850 \mathrm{pc})$ along the minor axis (Figure 4). To reduce the problems introduced by splitting features across slices, each slice is 5 !" 0 (85 pc) wide, and we overlap slices by half their width $\left(2{ }^{\prime \prime} 5,42 \mathrm{pc}\right)$. A sample $\mathrm{pV}$ diagram is shown in Figure 5 for the central slice, which runs along the major axis (offset $0 !^{\prime \prime} 0$ ). A complete set of $\mathrm{pV}$

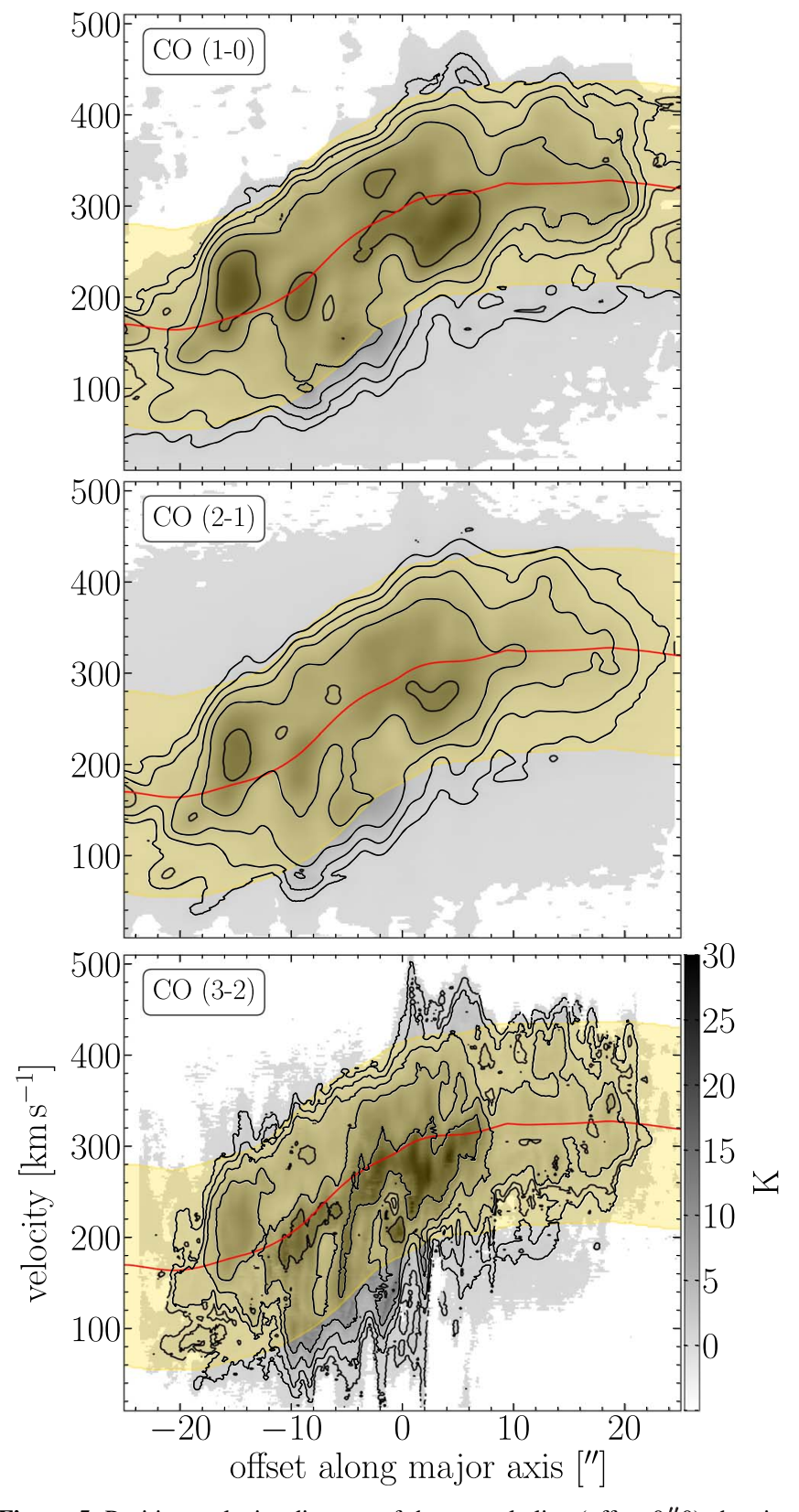

Figure 5. Position-velocity diagram of the central slice (offset $0 . / 0$ ) showing the construction of the disk/nondisk masks. The background images show the flux density above $5.0 \sigma$ for $\mathrm{CO}(1-0)$ (top), $\mathrm{CO}(2-1)$ (center), and $\mathrm{CO}(3-2)$ (bottom) on identical gray scales. Contours are drawn at $1,2,4,8$ and $16 \mathrm{~K}$. The central velocity for our model of the disk emission is illustrated by the red line. The golden-shaded area denotes the disk mask. Similar figures for other offsets are shown in Appendix C. Note that the different transitions have different angular resolutions.

diagrams is given in Appendix C. The resolution differences between our three transitions, a factor of $\sim 100$ in beam solid angle, are apparent in Figure 5. In the high angular resolution $\mathrm{CO}(3-2)$, small features with large line widths are common. These features are blurred out in the lower resolution $\mathrm{CO}(1-0)$ and $(2-1)$.

\subsection{Modeling the Disk}

We derive a model for the velocity of the disk component from the $\mathrm{CO}(1-0)$ observations using the kinematic fitting tool diskfit (Spekkens \& Sellwood 2007; Sellwood \& Sánchez 2010; 
Sellwood \& Spekkens 2015). Because the CO(1-0) observations cover the largest area among our observations, we use them to derive the model; the additional information provided by the $\mathrm{CO}(2-1)$ and/or $\mathrm{CO}(3-2)$ data is negligible in terms of the bulk motions of the gas. We obtain a $\mathrm{CO}(1-0)$ velocity field by computing the first moment of the cube after masking it at $20 \sigma$ $(1.26 \mathrm{~K})$, in order to represent the velocity of the bright emission. We show the details of the fit parameters and a comparison to the $\mathrm{CO}(1-0)$ velocity field in Appendix A.

In each $\mathrm{pV}$ slice, we use the velocity profile of the diskfit model to define the local disk velocity. We consider the $\mathrm{CO}$ emission to be consistent with the disk component of the emission when the velocity difference is within the local observed velocity range, $\Delta v$. This velocity range varies spatially and depends on the distance $x$ from the major axis, increasing toward the center due to the combined effects of higher intrinsic velocity dispersion and projection. For the success of this analysis, it is crucial that $\Delta v$ is broad enough to cover the observed velocity range of the disk but also narrow enough in order to not classify potential outflows as a disk. The definition of $\Delta v$ is thus a crucial source of uncertainty for the derived quantities. We parameterize the velocity range of the disk as

$$
\Delta v(x)=120 \exp \left(-\left(\frac{x}{2.5}\right)^{2}\right)+100,
$$

with $\Delta v$ in $\mathrm{km} \mathrm{s}^{-1}$ and $x$ in arcseconds. We find this empirical relation to fit the $\mathrm{pV}$ data best, and small variations of order 10\%-20\% already show a noticeable mismatch as discussed in Appendix B. The quality of this definition can be assessed from Figure 5 and Appendix $C$ : the velocity ranges are wide enough to include obvious emission of the disk but do not extend into the kinematically distinct features (potential outflows) that appear as spikes. This is most apparent for $\mathrm{CO}(3-2)$ as this line offers the highest spatial resolution. The effect of a $10 \%$ change in the velocity range $\Delta v$ corresponds to up to 0.1 dex variations in the derived quantities (see Appendix B).

\subsection{Selecting the Components}

We use the modeled velocity field and the $\Delta v$ relation together to define a "disk mask" over $\mathrm{ppV}$ space, corresponding to emission that is consistent with disk rotation. We show in Figure 5 the central $\mathrm{pV}$ slices for $\mathrm{CO}(1-0),(2-1)$, and (3-2). We show in Appendix $\mathrm{C}$ the complete set of $\mathrm{pV}$ slices.

Note that the $\mathrm{CO}$ emission extends beyond the disk mask. These extensions are not symmetric and due to nondisk gas and projection effects. At $\sim 78^{\circ}$ inclination, gas flowing perpendicular to the galaxy disk toward the south (negative slice offsets) is primarily approaching us and seen at lower velocities relative to the disk emission. Similarly, outflow emission toward the north is primarily at velocities higher than the disk emission. Consequently, emission in $\mathrm{pV}$ slices shifts from lower to higher velocities relative to the disk model when the offset from the major axis increases (see Figure 12 in Appendix C). We designed the disk mask to be wide enough to capture the disk emission but exclude the asymmetric component caused by outflows.

We define a "nondisk" mask that is the mathematical complement of the disk mask, with the addition of removing emission from known sources (portions of the spiral arms) that were not included in our model of the central disk and are not of interest for this analysis.

\subsection{Identifying Outflows in the Nondisk Component}

We identify three different types of structures in the nondisk component (Figure 6; contours in Figure 7 highlight these structures):

(1) Emission that is colocated with the central disk and bar in projection. This is visible as a ridge in $\mathrm{CO}(3-2)$ (inner contour in Figure 7) and also present but less apparent in $\mathrm{CO}(1-0)$ and $\mathrm{CO}(2-1)$. The structure is unlikely to be an outflow. It appears more likely to be an additional kinematic component of the disk/bar that is not included in the model we used for the separation. We therefore do not consider this gas to contribute to the total mass outflow rate.

(2) Emission associated with the so-called western superbubble, located to the west and north of the central starburst region (Sakamoto et al. 2006; Bolatto et al. 2013; shown by the western contour in Figure 7). This feature is already known to be kinematically distinct from the surrounding gas. Part of it is likely the base of the northern outflow cone (and giving rise to the NW streamers identified by Bolatto et al., for example), but it is difficult to know what portion of the emission should be associated with a net outflow. In our calculations below, we exclude this feature from the total outflow rate of NGC 253, although it likely has some contribution to the outflow.

(3) The remaining gas associated with the nondisk component is organized in small clumps along the edge of the disk region or beyond it. Some of this gas is not discernible as individual structures, particularly in the $\mathrm{CO}(1-0)$ and $\mathrm{CO}(2-1)$ cubes, perhaps due to the resolution but maybe also due to the excitation conditions, constituting extended regions with diffuse emission. Some of the emission is located in welldefined structures known to be part of the outflow, such as the SW streamer, which is apparent in all CO transitions.

In summary, the nondisk component consists of these three subcomponents: structures that we associate with a net "outflow," structures that are part of the "western superbubble," and structures that are colocated with the "disk." The latter is not associated with the outflow, while parts of the western superbubble may contribute to it. Below, we calculate properties for the two components, disk and nondisk, and its subcomponents individually where it is feasible to do so.

\section{Results}

The process described in the previous section allows us to estimate the properties of the galactic outflow and other structures. A 2D representation of the separated data cubes is shown in Figure 6 in the form of moment maps for integrated intensity (moment 0 ) and intensity-weighted velocity (moment 1) for all three CO transitions. Striping artifacts due to the $\mathrm{pV}$ cuts used in the separation method are present in the disk and nondisk components, visible as straight lines parallel to the major axis. This is primarily aesthetic. We tested their effects on the fluxes and derived velocities by varying the slice width and found them to be negligible.

\subsection{CO Luminosities}

We quantify in Table 2 the CO luminosities of the disk and nondisk components. We measure luminosities of $2.8 \times 10^{8} \mathrm{~K} \mathrm{~km}$ $\mathrm{s}^{-1} \mathrm{pc}^{2}, 2.3 \times 10^{8} \mathrm{~K} \mathrm{~km} \mathrm{~s}^{-1} \mathrm{pc}^{2}$, and $1.8 \times 10^{8} \mathrm{~K} \mathrm{~km} \mathrm{~s}^{-1} \mathrm{pc}^{2}$ for 
(a)

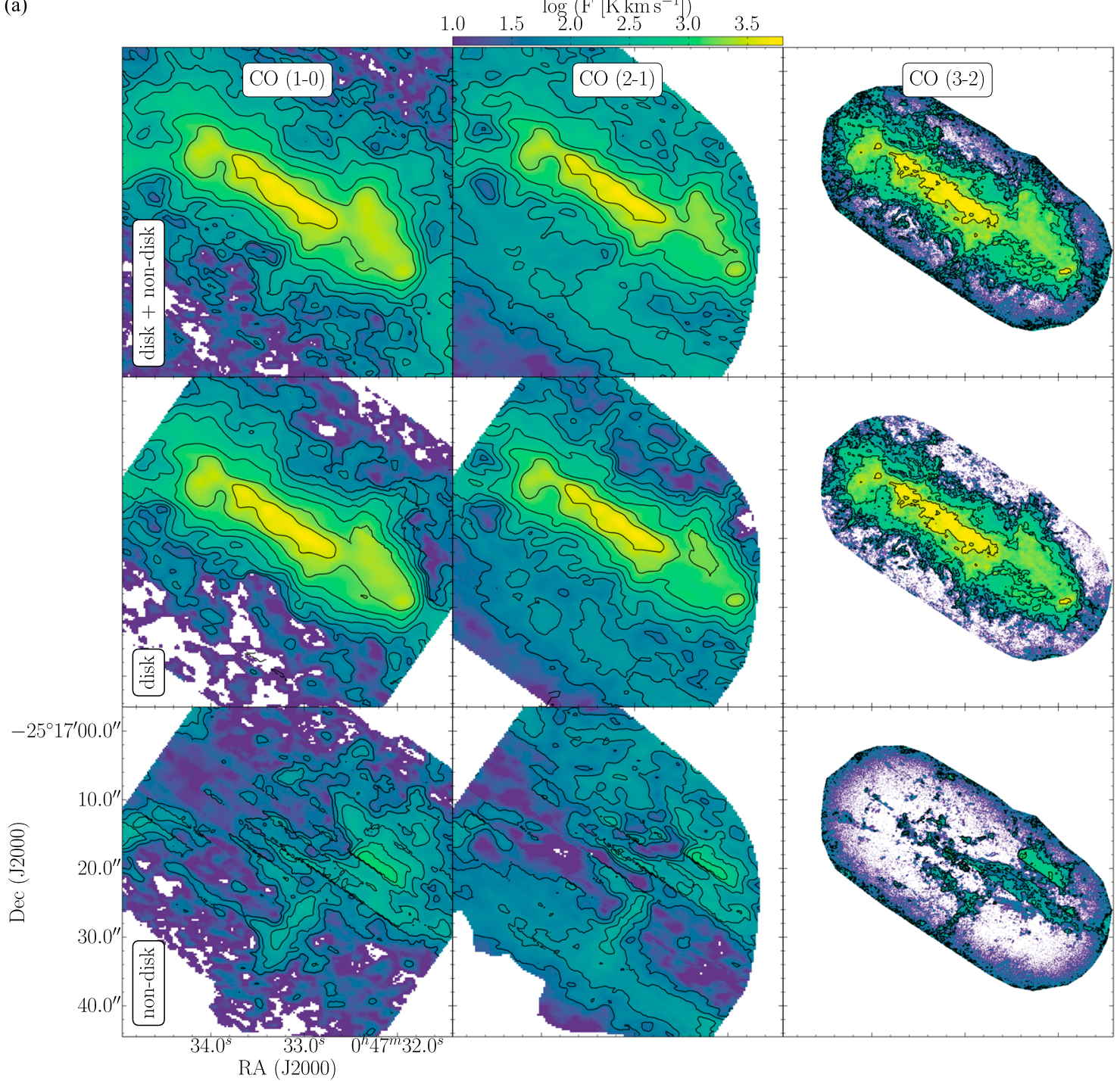

Figure 6. Comparison between the original moment maps and separated disk/nondisk components. (The outline of the maps is defined by the observed field of view and the square region considered for separating the kinematic components.) (a) Moment 0 (integrated intensity) of the original image (top), disk component (middle), and nondisk component (bottom). The logarithmic color scales are identical for all panels and chosen to also show the fainter nondisk component which saturates the inner regions of the disk. Contours are drawn at $\log \left(F\left[\mathrm{~K} \mathrm{~km} \mathrm{~s}^{-1}\right]\right)=1.7,2.0,2.3,2.6,2.9,3.2,3.5$; for clarity, only every other contour is drawn for $\mathrm{CO}(3-2)$. (b) Moment 1 (intensity-weighted velocity) of the original image (top), disk component (middle), and nondisk component (bottom). Contours are drawn at 150, 200, $\ldots, 350 \mathrm{~km} \mathrm{~s}^{-1}$. The noise edge visible in $\mathrm{CO}(3-2)$ is due to the primary beam correction required to derive accurate fluxes.

$\mathrm{CO}(1-0),(2-1)$, and (3-2), respectively, in the central disk of NGC 253. The nondisk component is, naturally, much fainter with luminosities of $\sim 4.2 \times 10^{7} \mathrm{~K} \mathrm{~km} \mathrm{~s}^{-1} \mathrm{pc}^{2}$ for $\mathrm{CO}(1-0), \sim 4.2 \times$ $10^{7} \mathrm{~K} \mathrm{~km} \mathrm{~s}^{-1} \mathrm{pc}^{2}$ for (2-1), and $\sim 4.2 \times 10^{7} \mathrm{~K} \mathrm{~km} \mathrm{~s}^{-1} \mathrm{pc}^{2}(3-2)$. These correspond to approximately $12.9 \%, 16.4 \%$, and $6.5 \%$ of the total luminosity. These luminosities are measured over the sliced area (see Figure 4) for which the coverage is not the same among the data sets. We therefore also measure luminosities integrated over the same spatial region, here defined as the overlap between the data sets. This overlap amounts to $885 \operatorname{arcsec}^{2}$ $\left(2.55 \times 10^{5} \mathrm{pc}^{2}\right)$. The luminosities in the overlap area are disk: $2.6 \times 10^{8} \mathrm{~K} \mathrm{~km} \mathrm{~s}^{-1} \mathrm{pc}^{2}, \quad 2.1 \times 10^{8} \mathrm{~K} \mathrm{~km} \mathrm{~s}^{-1} \mathrm{pc}^{2}$, and $1.8 \times$ $10^{8} \mathrm{~K} \mathrm{~km} \mathrm{~s}^{-1} \mathrm{pc}^{2}$; nondisk: $2.6 \times 10^{7} \mathrm{~K} \mathrm{~km} \mathrm{~s}^{-1} \mathrm{pc}^{2}, \quad 2.4 \times$ $10^{7} \mathrm{~K} \mathrm{~km} \mathrm{~s}^{-1} \mathrm{pc}^{2}$, and $1.2 \times 10^{7} \mathrm{~K} \mathrm{~km} \mathrm{~s}^{-1} \mathrm{pc}^{2}$ for $\mathrm{CO}(1-0)$, (2-1), and (3-2), respectively.

An interesting result coming out of our decomposition is that not all of the material we identify as "outflow" is in well-defined structures such as the streamers identified by Bolatto et al. (2013).
Correctly estimating the outflow rate requires also accounting for a diffuse extended component.

It is important to compare our fluxes to measurements in the literature. Mauersberger et al. (1996) found a $\mathrm{CO}(2-1)$ luminosity of $1.2 \times 10^{6} \mathrm{~K} \mathrm{~km} \mathrm{~s}^{-1} \operatorname{arcsec}^{2}$, which translates ${ }^{13}$ to $3.5 \times 10^{8} \mathrm{~K} \mathrm{~km} \mathrm{~s}^{-1} \mathrm{pc}^{2}$ or 1.3 times our measurement. Their observations cover $80^{\prime \prime} \times 60^{\prime \prime}$, an area similar to our $\mathrm{CO}(1-0)$ observations (but $\sim 4$ times larger than the area of our $\mathrm{CO}(3-2)$ observations). For the outflow $\mathrm{CO}(1-0)$ luminosity, Bolatto et al. (2013) derived an estimate of $2.0 \times 10^{7} \mathrm{~K} \mathrm{~km} \mathrm{~s}^{-1} \mathrm{pc}^{2}$ by summing over individual identified molecular outflow features. This includes flux from the "superbubble" component, so it is probably better compared to the sum of our "outflow" and "superbubble" components of $\sim 3.6 \times 10^{7} \mathrm{~K} \mathrm{~km} \mathrm{~s}^{-1} \mathrm{pc}^{2}$. Given the large methodological differences and the importance

\footnotetext{
13 Adjusting from the distance $D=2.5 \mathrm{pc}$ used by Mauersberger et al. to the $D=3.5 \mathrm{pc}$ assumed here.
} 


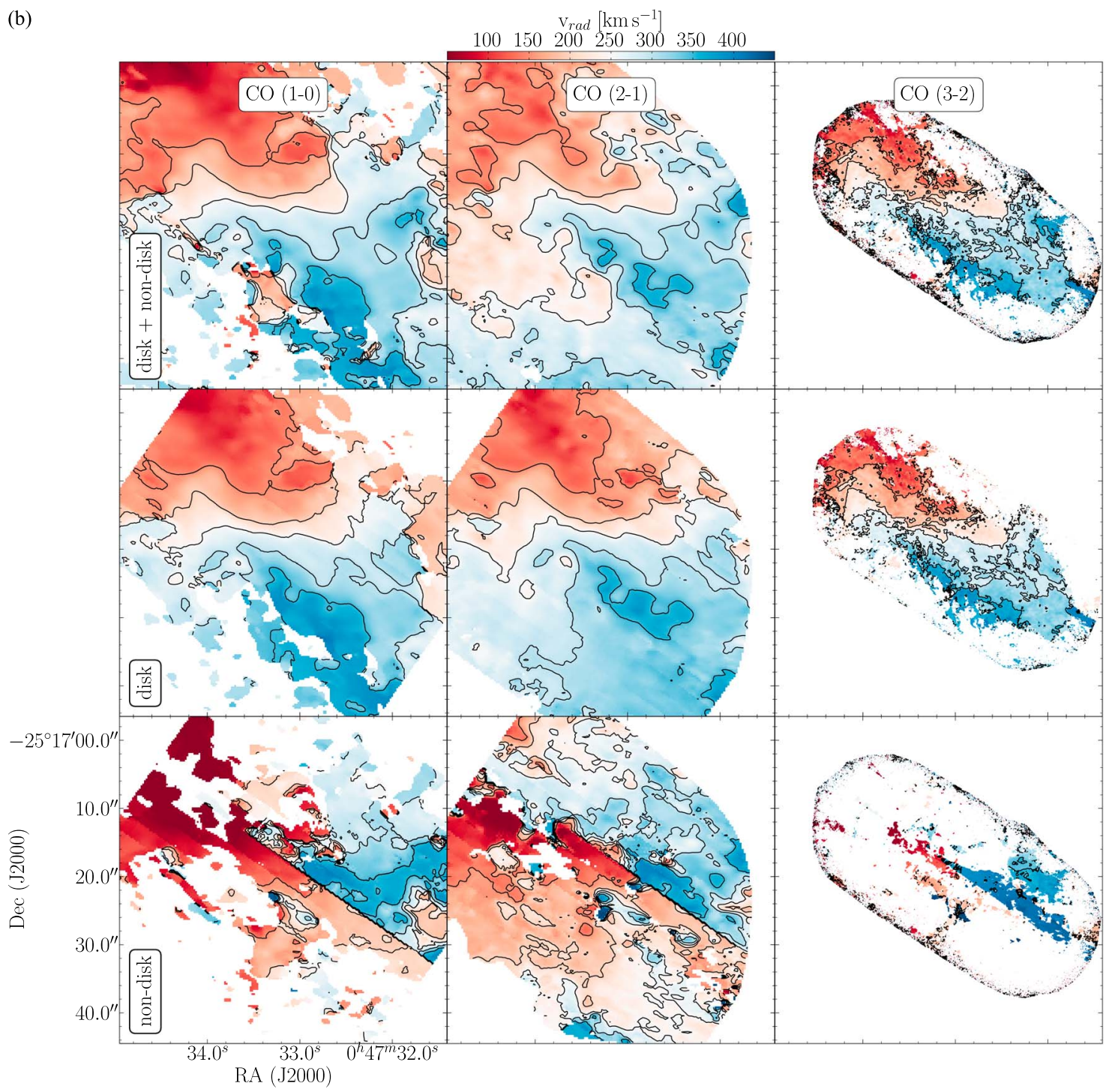

Figure 6. (Continued.)

of the diffuse emission, these numbers are in reasonable agreement.

\subsection{Masses of Components}

The total gas mass $M$ is estimated from the $\mathrm{CO}$ line luminosity, using the conversion factor $X_{\mathrm{CO}}=0.5 \times 10^{20}\left(\mathrm{~K} \mathrm{~km} \mathrm{~s}^{-1}\right)^{-1}$ $\mathrm{cm}^{-2}$ corresponding to $\alpha_{\mathrm{CO}}=1.1 M_{\odot}\left(\mathrm{K} \mathrm{km} \mathrm{s}^{-1} \mathrm{pc}^{-1}\right)^{-1}$ discussed by Leroy et al. (2015a) for the central starburst region. This value accounts for the effects of moderate optical depth, highvelocity dispersion, and warm gas temperatures that are likely to dominate the central regions of NGC 253. The masses we report include the contribution of helium to the total mass. To compute masses using the $\mathrm{CO}(2-1)$ and $\mathrm{CO}(3-2)$ transitions, we assume typical line ratios of $r_{21}=0.80$ and $r_{31}=0.67$ relative to $\mathrm{CO}(1-0)$ as implied by Zschaechner et al. (2018). Note that we do not measure line ratios from the images but adopt a uniform factor to keep the mass measurements from the three observed CO lines independent.

Table 2 lists the masses corresponding to the disk and the nondisk components. Uncertainty in the mass estimates arises primarily from the assumed conversion factor and the apportioning of emission among the different components. The calibration uncertainty for the flux measurements is $\sim 10 \%-15 \%$ for the ALMA observations. Overall, we adopt a systematic error of factor $\sim 2$ for the the derived masses.

The molecular masses derived from the three $\mathrm{CO}$ transitions are very similar. They match within $10 \%$ for the disk component, and within $50 \%$ for the nondisk component. We estimate the total gas mass in the center of NGC 253 to be $\sim 3.5 \times 10^{8} M_{\odot}$ (adding the disk and nondisk components), with estimates in the range of $3.1-3.6 \times 10^{8} M_{\odot}$ for the different transitions. About $85 \%$ of the total mass is in the disk component. The masses estimated in the nondisk components using $\mathrm{CO}(1-0)$ and $\mathrm{CO}(2-1)$ are fairly similar at $4.5 \times 10^{7} M_{\odot}$ and $6.1 \times 10^{7} M_{\odot}$, whereas in $\mathrm{CO}(3-2)$ we detect a lower mass, $2.0 \times 10^{7} M_{\odot}$, a consequence of the lower luminosity. The nondisk masses are primarily contributed by the outflow component ( $\sim 50 \%)$. About $20 \%-30 \%$ of mass is in the western superbubble and $12 \%-30 \%$ is cospatial with the disk but kinematically distinct.

It is important to compare these mass estimates to previous results for the total molecular gas mass in NGC 253, noting that 


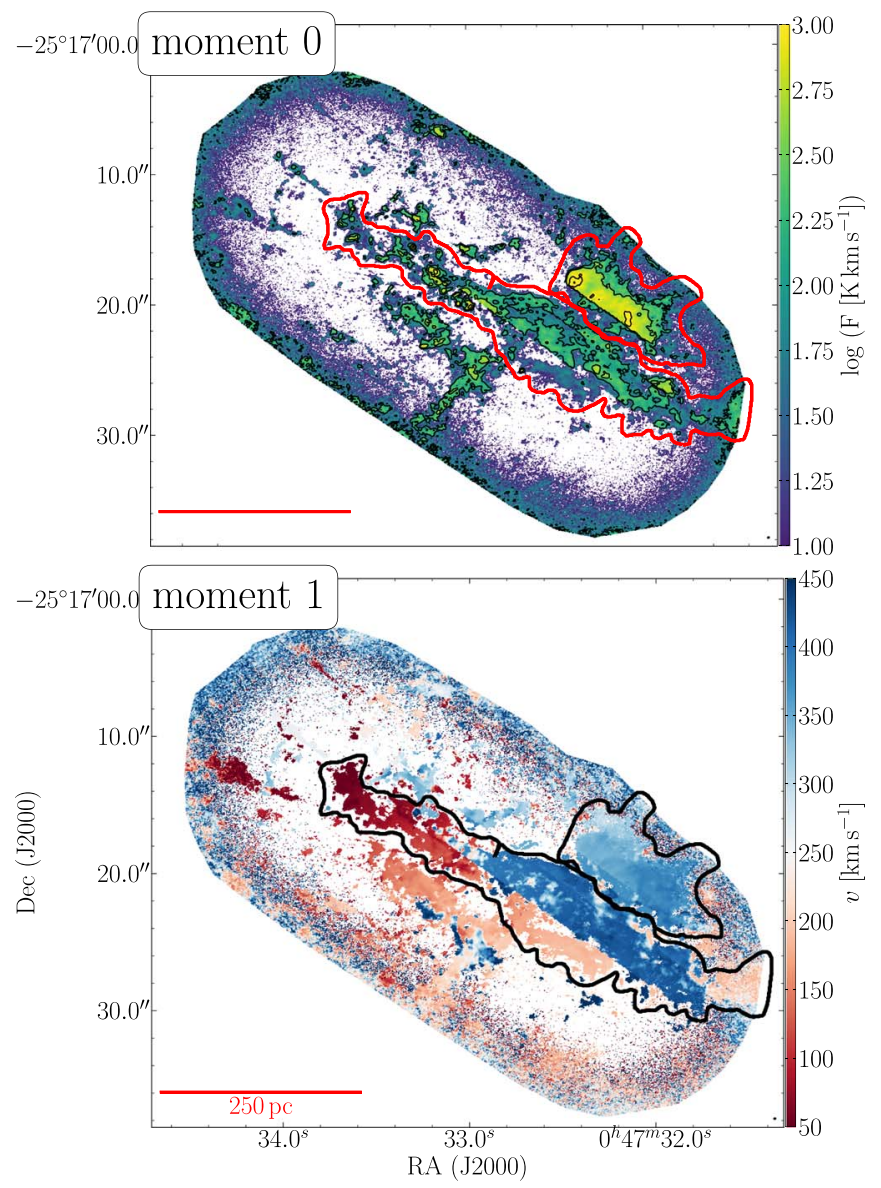

Figure 7. A zoom-in on the nondisk component of $\mathrm{CO}(3-2)$ (the bottom left panels in Figures 6(a) and (b)). Top: moment 0 (integrated intensity) with contours at $\log \left(F\left[\mathrm{~km} \mathrm{~s}^{-1}\right]\right)=2.0,2.5,3.0$. Bottom: moment 1 (intensityweighted velocity). The thick contours show the regions discussed in the text (Section 4.3): gas that is kinematically not consistent with disk rotation but cospatial with the disk in projection, and the western superbubble to the northwest of the disk.

our analysis covers the central $45^{\prime \prime} \times 25^{\prime \prime}(750 \mathrm{pc} \times 400 \mathrm{pc})$. Toward the east, $\sim 10 \%$ of the known molecular gas close to the center is not covered by our $\mathrm{CO}(3-2)$ observations and thus not considered in this analysis. The agreement with previous measurements is very good. Mauersberger et al. (1996) reported a mass of $1.3 \times 10^{8} M_{\odot}$ over a similar area $\left(80^{\prime \prime} \times 60^{\prime \prime}\right.$ in the center of NGC 253), but this was based on a different distance and $X_{\mathrm{CO}}$. After correcting for those differences, their luminosity corresponds to $4.2 \times 10^{8} M_{\odot}$, consistent with our measurement. Using the same distance and the same 1-0 observations, Leroy et al. (2015a) measured a molecular mass of $3.5 \times 10^{8} \mathrm{M}_{\odot}$. Pérez-Beaupuits et al. (2018) reported a total gas mass of $4.5 \times 10^{8} M_{\odot}$ derived from the submillimeter dust spectral energy distribution, which is very consistent with our result given the very different methodologies.

No estimates in the literature separate the "disk" and "nondisk" components as we do above. Previous estimates of the outflowing mass range from a lower limit of $6.6 \times 10^{6} M_{\odot}$ calculated for the optically thin limit (Bolatto et al. 2013), to $2-4 \times 10^{7} M_{\odot}$, when accounting for optical depth (Zschaechner et al. 2018). Because we identify an outflowing mass of $\sim 5 \times 10^{7} M_{\odot}$, the agreement with the latter estimate is fairly good. Note, however, that these studies derive the outflowing mass from individual features rather than using the $\mathrm{pV}$ information as we do here in a systematic way.

\subsection{Mass Outflow Rate}

Mass flow rate is defined as the flux of mass per unit time through a surface. In our case, we are interested in the flow of molecular gas mass through a virtual closed surface around the center of NGC 253 at a given distance. Note that an individual outflow feature observed over a certain length, such as the SW streamer, can develop in at least two ways: as the distance of an outflow from its origin corresponds to time since ejection times velocity, continuously outflowing gas results in extended streaming structures. Gas ejected at a single ejection event in the past with a distribution of ejection velocities, on the other hand, will also result in an extended streamer. In reality, gas will be ejected with a distribution of velocities at a varying rate over a period of time, and in order to interpret the measurements, we need to make some simplifying assumptions. We chose two edge cases to span the range of different interpretations: (1) the gas does not experience accelerations after being launched (however, see Walter et al. 2017), and (2) that the gas outflow rate is approximately constant with time. For all calculations, however, we assume that the projected direction of flow is perpendicular to the central plane of the bar and that $\mathrm{CO}$ emission traces the mass with a constant conversion factor.

We compute the outflow rate both as a function of distance and as a function of time. If we assume that the mass outflow rate has been approximately constant over the lifetime of the starburst, for example, a diminishing outflow rate as a function of distance would suggest that gas is either launched with or somehow develops a distribution of velocities. Conversely, if we assume that the present-day velocity has been constant since the gas was ejected, we can derive a history of the mass outflow rate as a function of time and account for a variable mass outflow rate. Both interpretations are equally, but not simultaneously, valid.

For the detailed calculation of the mass outflow rate, we proceed as follows. The mass outflow rate is $\dot{M}=M t^{-1}$ with mass $M$ and relevant timescale $t$. For each image element $i$ (3D pixel or sometimes also called voxel), we calculate the outflow rate $\dot{m}_{i}$ of the gas that was ejected at time $t_{\text {eject, } i}$ over the time interval $\Delta t_{\text {cross }, i}$, as the ratio of mass of the pixel $m_{i}$ to the pixel-crossing interval $t_{\mathrm{cross}, i}$. The ejection time and pixelcrossing interval are functions of the outflow velocity $v_{i}$ and the distance $s_{i}$ between the current pixel position and the launching site, and the pixel size in the direction of the flow $\Delta s$, respectively. We therefore compute

$$
\begin{aligned}
\Delta t_{\text {cross }, i} & =\frac{\Delta s}{v_{i}} \\
\dot{m}_{i}\left(t_{\text {eject }, i}\right) & =\frac{m_{i}}{\Delta t_{\text {cross }, i}} \\
t_{\text {eject }, i} & =\frac{s_{i}}{v_{i}},
\end{aligned}
$$

obtaining a mass outflow rate $\dot{m}_{i}$, a distance $s_{i}$, and an ejection time $t_{\text {eject }, i}$ for each pixel in the "outflow" component. Note that this approach takes the 3D phase space information into account by treating pixels independently. Typically, a sight line shows multiple pixels with emission at different velocities that all contribute an outflow rate with their respective mass, distance, and velocity. We then bin the outflow rates $\dot{m}_{i}$ by ejection time $t_{\text {eject, } i}$ and integrate over the time range $\left[T_{1}, T_{2}\right]$ to 
Table 2

Results of Separating Disk from Nondisk Emission in NGC 253

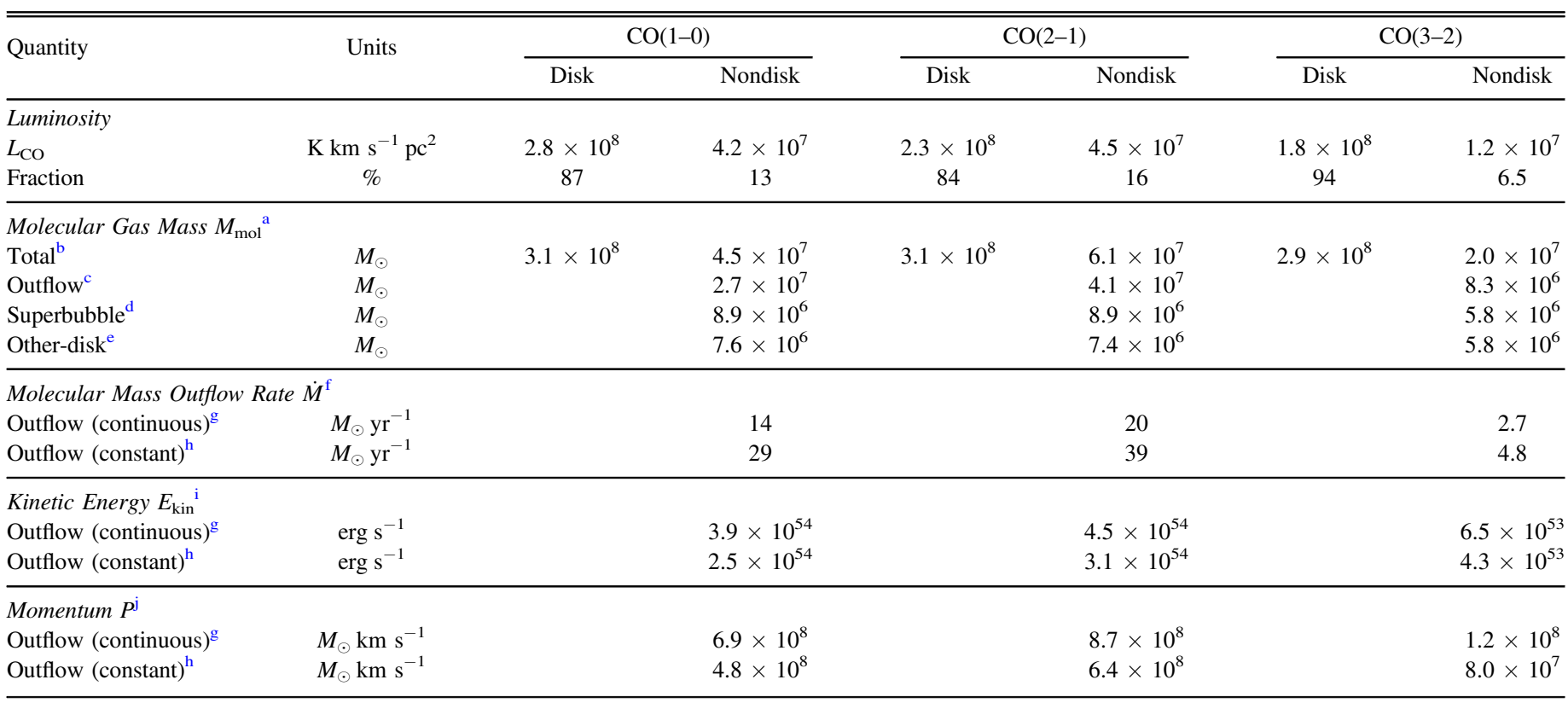

Notes. Uncertainties for these quantities are discussed in the corresponding subsections of Section 4. Sources of error are discussed and quantified in the respective subsections of Section 4.

${ }^{\text {a }}$ Molecular gas mass derived using a conversion factor for $\mathrm{CO}(1-0)$ emission of $X_{\mathrm{CO}}=0.5 \times 10^{20}\left(\mathrm{~K} \mathrm{~km} \mathrm{~s}^{-1}\right)^{-1}$, including the contribution of helium, and assuming CO brightness temperature line ratios of $r_{21}=0.80$ and $r_{31}=0.67$ for $\mathrm{CO}(2-1)$ and $\mathrm{CO}(3-2)$ relative to $\mathrm{CO}(1-0)$.

${ }^{\mathrm{b}} \mathrm{CO}$ line luminosity of all emission considered consistent with disk rotation (disk) and not consistent with disk rotation (nondisk), respectively.

${ }^{c}$ Nondisk excluding the western superbubble and the gas that is cospatial with the projected disk.

${ }^{\mathrm{d}}$ Nondisk emission belonging to the western superbubble as defined by Sakamoto et al. (2006).

${ }^{\mathrm{e}}$ Nondisk gas that is cospatial with the disk in projection. See Section 4.3 for the definition.

${ }^{\mathrm{f}}$ Deprojected molecular mass outflow rate. Fiftieth percentile best estimate assuming a flat distribution of outflow inclinations for the unknown geometry.

${ }^{g}$ Outflowing gas as defined by note (c) under the assumption of continuous mass ejection without accelerations to the gas after ejection.

h Outflowing gas as defined by note (c) under the assumption of approximately constant starting mass outflow rate over the lifetime of the starburst.

${ }^{\mathrm{i}}$ Deprojected kinetic energy of the molecular gas. Fiftieth percentile best estimate assuming a flat distribution of outflow inclinations for the unknown geometry.

${ }^{\mathrm{j}}$ Deprojected momentum of the molecular gas. 50th percentile best estimate assuming a flat distribution of outflow inclinations for the unknown geometry.

obtain the average outflow rate in this time interval,

$$
\dot{M}\left(T_{1}, T_{2}\right)=\frac{\sum_{i} \dot{m}_{i}\left(T_{1}<t_{\text {eject }, i}<T_{2}\right) \Delta t_{\text {cross }, i}}{T_{2}-T_{1}}
$$

Similarly, binning by distance results in the average outflow rate at a given distance,

$$
\dot{M}\left(D_{1}, D_{2}\right)=\frac{\sum_{i} \dot{m}_{i}\left(D_{1}<s_{i}<D_{2}\right) \Delta s}{D_{2}-D_{1}} .
$$

Performing binning on a sequence of time intervals yields the outflow rate history, while binning in distance tells us how far from the launching site a given fraction of the mass is able to escape.

Calculating velocity $v$ and distance $s$ requires knowledge about the geometry and origin of each outflowing gas parcel. The simplest assumption, used here, is that on average, outflows are launched in the plane of the central region of the galaxy, which corresponds to launching on the major axis. The distance $s$ is thus the projected distance to the major axis on the edge of an outflow cone with a given opening angle. Note that the outflow originates from an extended region in the disk and the term cone thus refers to a cutoff cone (called a frustum in geometry). The velocity $v$ is the velocity difference between the launching site and current velocity of the outflow parcel, i.e., the velocity difference over the distance $s$. The velocities of both the launching site and the projected distance are uncertain. The velocity changes by $\pm 25 \mathrm{~km} \mathrm{~s}^{-1}$ when an outflow originates from the northern/southern edge of the observed CO disk (above/below the plane), while the projected distance traveled by the gas changes by \pm 1 ". 25 ( $\pm 20 \mathrm{pc}$ ).

Distance $s$, ejection timescale $t_{\text {eject }}$, and pixel-crossing timescale $t_{\text {cross }}$ are measured as projected quantities that need to be deprojected to account for the outflow geometry. The bright molecular streamers (the SW and SE streamers) seem to lie at the edge of the ionized outflow cone with a $\sim 60^{\circ}$ opening angle (Bolatto et al. 2013). Assuming that all molecular outflows are along this cone and that the axis of the cone is oriented perpendicular to the disk $\left(i=78^{\circ}\right)$, the range of the effective inclination of outflowing gas can be anywhere between $\theta=48^{\circ}$ and $\theta=108^{\circ}$. Deprojected velocity, $v_{\text {depro }}=$ $v_{\mathrm{obs}} / \sin \theta$, and distance, $s_{\mathrm{depro}}=s_{\mathrm{obs}} / \cos \theta$, have a direct effect on the deprojected outflow rate, $\dot{m}_{\text {depro }}=\dot{m}_{\text {obs }} \tan \theta$, and also on the inferred time and distance evolution of the outflow rate. We use a Monte Carlo approach to derive the errors introduced by deprojection, assuming that the outflow direction has an equal probability of being in any direction along the surface of the outflow cone.

Figure 8 shows the molecular mass outflow rate as a function of time or distance, corresponding to the two alternative 

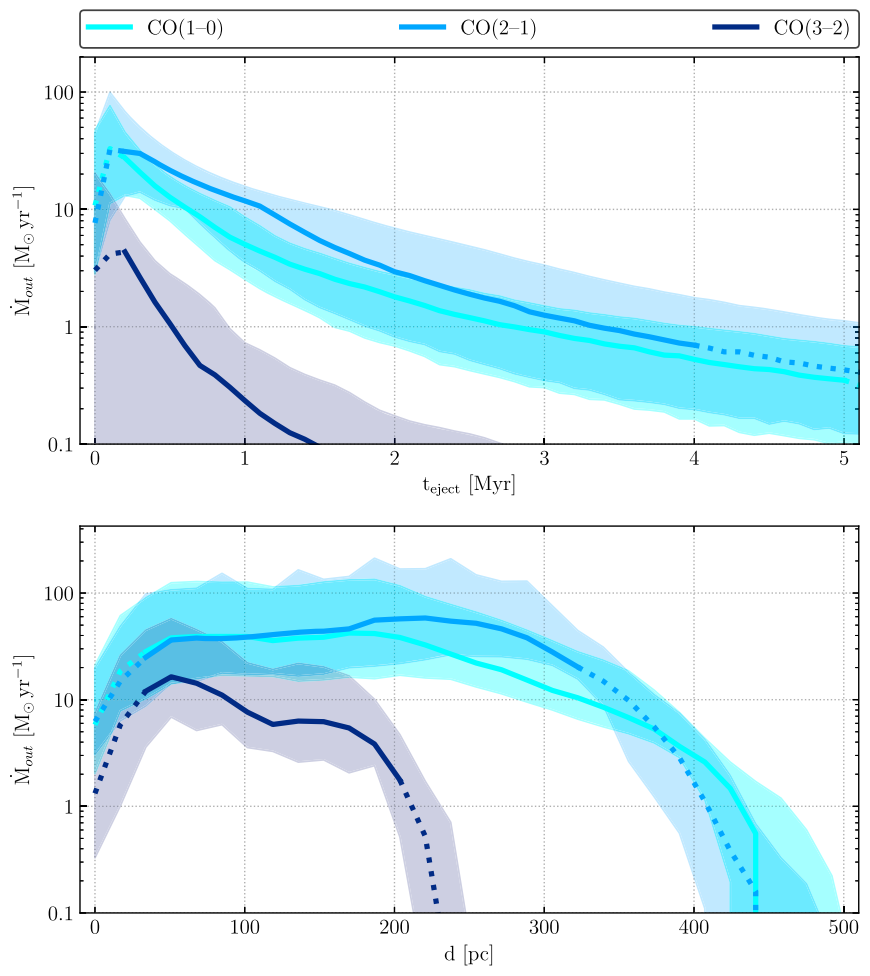

Figure 8. Deprojected molecular mass outflow rate averaged over 0.1 Myr as a function of time since ejection (top) and as a function of deprojected distance between outflow and launching site (bottom). The top panel implicitly assumes continuous mass ejection without accelerations to the gas after ejection, while the lower panel assumes an approximately constant starting mass outflow rate over the lifetime of the starburst. The shaded area indicates approximate errors (16th-84th percentile), which are dominated by uncertainties in the deprojection geometry. Dotted lines represent the ranges where confusion with gas in the disk occurs and where the limited field of view affects the completeness.

interpretations we discuss above: a flow where the distribution of material is interpreted as resulting from the history of the mass outflow rate (top panel) and one where we show the mass outflow rate as a function of distance, which under the assumption of a constant outflow rate over the last several megayears can be interpreted as the efficiency of ejection to a given distance (bottom panel). Indeed, for an outflow with a distribution of velocities, the slower material will not travel as far in a given time, and neither will it escape the galaxy if it does not have a high-enough velocity. Close to the starburst region (or at small times since ejection), the mass outflow rate drops to zero, because it becomes increasingly difficult to separate the "outflow" component from the "disk" component. At large values of distance or time, it also drops to zero, due to a decreasing amount of outflowing molecular material detected far from the starbursts (and the fact that the observations have a limited field of view). The constant outflow rate out to $\sim 300 \mathrm{pc}$ is in tension with Kim \& Ostriker (2018), who find a steeply dropping "cold" $(T<5050 \mathrm{~K})$ component in their TIGRESS simulation. Within $400 \mathrm{pc}$, they find the average mass-loading factor to drop by two orders of magnitude. It is unlikely that the SFR in NGC 253 has increased by two orders of magnitude within the past 1-2 Gyr, which would alter the observed constant outflow rate profile to be consistent with the Kim \& Ostriker (2018) simulation. Note, however, that their simulation recreates solar neighborhood-like conditions instead of a starburst. A direct comparison may thus not be possible.
Our data show that the average outflow rates within $20^{\prime \prime}$ (340 pc) from the major axis are $29 M_{\odot} \mathrm{yr}^{-1}\left({ }_{-0.35}^{+0.48} \mathrm{dex}\right)$, $39 M_{\odot} \mathrm{yr}^{-1}\left({ }_{-0.34}^{+0.49} \mathrm{dex}\right)$, and $\left.4.8 M_{\odot} \mathrm{yr}^{-1}{ }_{-0.39}^{+0.50} \mathrm{dex}\right)$ for $\mathrm{CO}(1-0),(2-1)$, and (3-2), respectively. Similarly, within the past $1.0 \mathrm{Myr}$, the average outflow rates are $14 M_{\odot} \mathrm{yr}^{-1}\left({ }_{-0.29}^{+0.25} \mathrm{dex}\right)$, $20 M_{\odot} \mathrm{yr}^{-1}\left(_{-0.37}^{+0.27} \mathrm{dex}\right)$, and $\left.2.7 M_{\odot} \mathrm{yr}^{-1}{ }_{-0.56}^{+0.22} \mathrm{dex}\right)$ for $\mathrm{CO}(1-0)$, $(2-1)$, and (3-2), respectively. The uncertainties, indicated by the 16th-84th percentiles in the Monte Carlo described above, are substantial at a factor of 2-3. Real systematic uncertainties are even larger, because there can be conversion of molecular into atomic material (see Leroy et al. 2015b), or in general variations in the CO-to- $\mathrm{H}_{2}$ conversion.

Note that the average outflow rates quoted above differ between the two representations, with the median outflow rate as a function of distance being about twice as high as a function of time. The outflowing mass is identical in both cases, and the difference arises solely from binning. Comparing between lines, it is apparent that as measured in $\mathrm{CO}(3-2)$, the outflow rate is roughly one order of magnitude lower than for the lower two transitions. This is a direct consequence of the lower mass detected in $\mathrm{CO}(3-2)$ and the smaller field of view of those observations. The $\mathrm{CO}(3-2)$ observations cover only $\sim 12$ !"5 ( $\sim 210 \mathrm{pc}$ projected) above/below the disk and thus miss significant amounts of nondisk gas. Their lower surface brightness sensitivity means we also fail to detect a diffuse nondisk component, as we see in the two lower lines. The measurements in $\mathrm{CO}(3-2)$ should thus be interpreted as a lower limit, and in that sense they are consistent with those for the lower two transitions.

Overall, the deprojected total mass outflow rate in the starburst of NGC 253 is most likely in the range $\sim 14-39 M_{\odot} \mathrm{yr}^{-1}$ as derived from $\mathrm{CO}(1-0)$ and $\mathrm{CO}(2-1)$ with $\sim 0.4$ dex uncertainty. The large spread arises due to different interpretations of the kinematics of the observed gas while the errors are due to unknown geometry. The majority of this outflow rate is contributed by massive outflows alongside the disk like the $\mathrm{SW} / \mathrm{SE}$ streamers, with a significant contribution by diffuse molecular gas.

The present-day SFR in the central region of NGC 253 is 1.7-2.8 $M_{\odot} \mathrm{yr}^{-1}$, derived from radio continuum and farinfrared measurements (Ott et al. 2005; Bendo et al. 2015; Leroy et al. 2015a). This results in a mass-loading factor $\eta=\dot{M}_{\text {out }} / \dot{M}_{\mathrm{SFR}}$ in the range $\eta \sim 5.4-23.5$. Note that this is for gas ejected as far as $340 \mathrm{pc}$. We do not currently know what fraction of the gas makes it to the far regions of the halo or reaches escape velocity from the system. Theoretical works suggest that most of the molecular outflow will not escape but rain back down on the galaxy (e.g., Shapiro \& Field 1976 up to recent work by Kim \& Ostriker 2018 or Tollet et al. 2019). In our data, no gas reaches the escape velocity of $v_{\mathrm{esc}}=500 \mathrm{~km} \mathrm{~s}^{-1}$ (Walter et al. 2017). The uncertainty on $v_{\text {esc }}$ is substantial, so allowing a factor of 2 is still plausible. At $v_{\mathrm{esc}}=250 \mathrm{~km} \mathrm{~s}^{-1}$, the fraction of gas above $v_{\text {esc }}$ by mass is $0.5 \%, 0.5 \%$, and $6.0 \%$ for $\mathrm{CO}(1-0), \mathrm{CO}(2-1)$, and $\mathrm{CO}(3-2)$, respectively. The mismatch between the lower transitions and $\mathrm{CO}(3-2)$ implies that some high-velocity gas can be found on small scales that is blurred out in the low-resolution observations.

This estimate of the molecular mass outflow rate is higher than the lower limit found by Bolatto et al. (2013) for optically thin emission. Zschaechner et al.'s (2018) analysis of the CO line ratios in the SW streamer shows that the emission there is optically thick, which the authors used to 

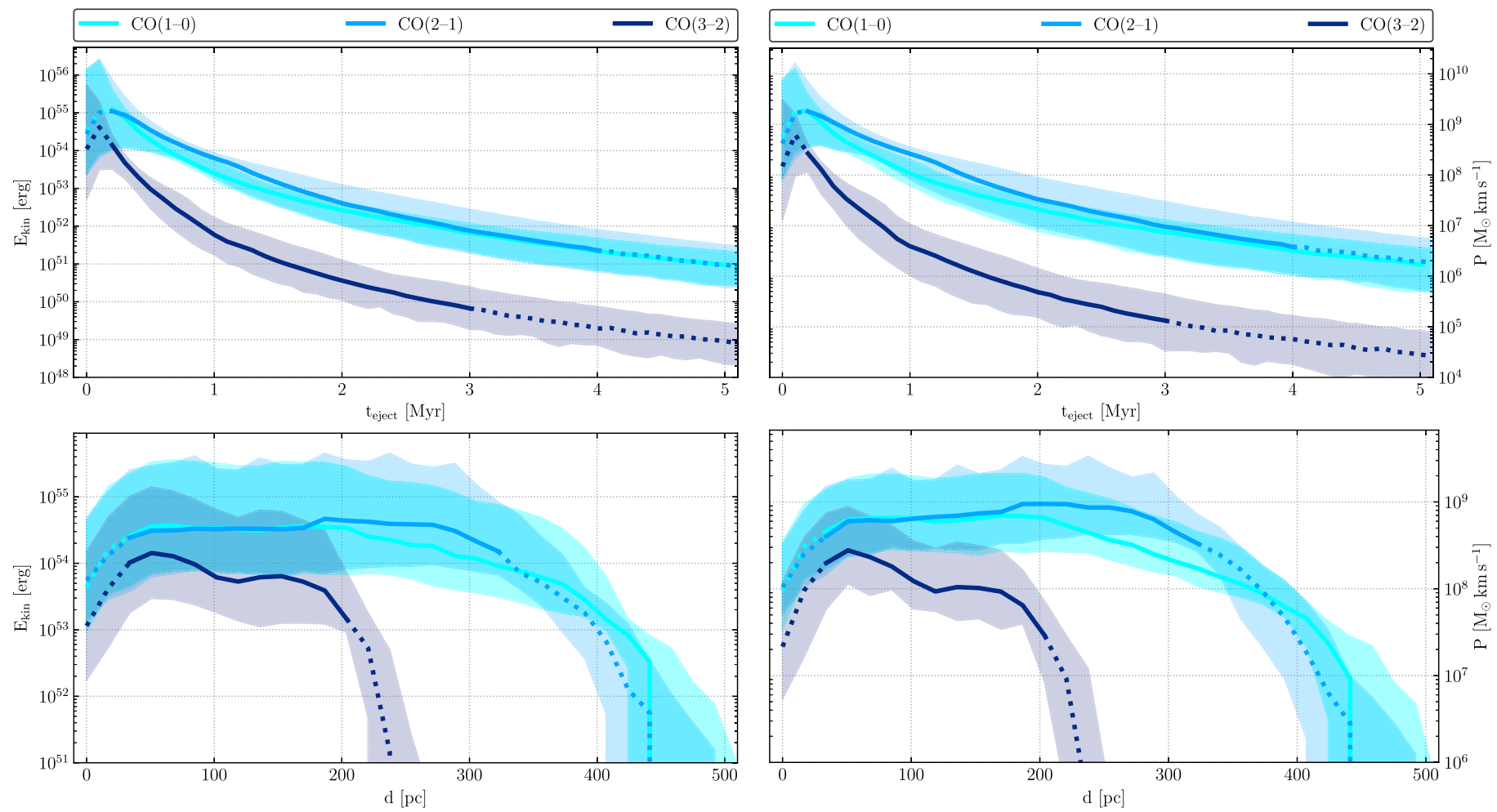

Figure 9. Deprojected kinetic energy (left) and deprojected momentum (right) of the molecular outflow averaged over 0.1 Myr as a function of time since ejection (top) and as a function of deprojected distance between the outflow and launching site (bottom). The top panel implicitly assumes continuous mass ejection without accelerations to the gas after ejection, while the lower panel assumes an approximately constant starting mass outflow rate over the lifetime of the starburst. The shaded area indicates approximate errors (16th-84th percentile), which are dominated by uncertainties in the deprojection geometry. Dotted lines represent the ranges where confusion with gas in the disk occurs and where the limited field of view affects the completeness.

rescale the Bolatto et al. (2013) measurements, finding a NGC 253 galactic outflow rate of $25-50 M_{\odot} \mathrm{yr}^{-1}$. The result presented here, a mass outflow rate of $\sim 14-39 M_{\odot} \mathrm{yr}^{-1}$, is consistent with this number using an independent and a more complete methodology than the original work.

From $\mathrm{H} \alpha$ observations by Westmoquette et al. (2011), we can estimate the ionized outflow rate to be $\sim 4 M_{\odot} \mathrm{yr}^{-1}$ using their ionized mass $\left(M=10^{7} M_{\odot}\right)$ and typical velocity $\left(200 \mathrm{~km} \mathrm{~s}^{-1}\right)$ at a mean deprojected distance $(510 \mathrm{pc})$. X-ray observations yield comparable values. Strickland et al. (2000) found an upper limit of $2.2 M_{\odot} \mathrm{yr}^{-1}$ assuming a standard outflow velocity of $3000 \mathrm{~km} \mathrm{~s}^{-1}$. The upper limit reported in Strickland et al. (2002) translates to $2.3 M_{\odot} \mathrm{yr}^{-1}$ when assuming $3000 \mathrm{~km} \mathrm{~s}^{-1}$ outflow velocity and a reasonable $10 \%$ filling factor. These estimates scale linearly with the unknown velocity and also depend on the unknown metallicity and filling factor in the outflow. Estimates of the outflow rate in neutral gas are not known in the literature but are arguably at a similar level. The molecular phase thus clearly dominates the mass budget in the outflow close to the disk as found in other galaxies (e.g., M82, Leroy et al. 2015b; and simulations, e.g., Kim \& Ostriker 2018).

\subsection{Outflow Energy and Momentum}

Similar to the mass outflow rate, energy and momentum can be calculated as a function of time and distance, which is shown in Figure 9. In Equations (5) and (6), the molecular outflow rate $\dot{m}_{i}$ is replaced by kinetic energy $E_{\mathrm{kin}, i}=\frac{1}{2} m_{i} v_{i}^{2}$ or momentum $P_{i}=m_{i} v_{i}$. As with the outflow rate, the dominant sources of error are the uncertainty in the launching site and the geometry for which we Monte Carlo the errors as described before. Our median estimate with 16th and 84th percentile uncertainties are given below and in Table 2 .

The kinetic energy in the outflow integrated over the past 1.0 Myr is $3.9 \times 10^{54} \mathrm{erg}\left({ }_{-0.75}^{+0.91} \mathrm{dex}\right)$ in $\mathrm{CO}(1-0), 4.5 \times 10^{54} \mathrm{erg}$ $\left({ }_{-0.80}^{+0.94}\right.$ dex $)$ in $\mathrm{CO}(2-1)$, and $6.5 \times 10^{53}$ erg $\left({ }_{-0.83}^{+0.58} \mathrm{dex}\right)$ for $\mathrm{CO}(3-2)$. Within $20^{\prime \prime}(340 \mathrm{pc})$, the kinetic energies amount to $2.5 \times 10^{54} \mathrm{erg}\left({ }_{-0.65}^{+0.96} \mathrm{dex}\right)$ in $\mathrm{CO}(1-0), 3.1 \times 10^{54} \mathrm{erg}\left({ }_{-0.65}^{+0.96} \mathrm{dex}\right)$ in $\mathrm{CO}(2-1)$, and $4.3 \times 10^{53} \mathrm{erg}\left({ }_{-0.64}^{+0.98} \mathrm{dex}\right)$ for $\mathrm{CO}(3-2)$. For the reasons described above, the $\mathrm{CO}(3-2)$ measurement is a lower limit, thus the results for the lower transitions are consistent.

NGC 253 does not appear to host an energetically important AGN, and the outflow is driven by the starburst. It is interesting then to compare our results for the kinetic energy to the energy released by the starburst. We assume the current SFR of $\sim 2.8 M_{\odot} \mathrm{yr}^{-1}$ in the central region (Ott et al. 2005; Bendo et al. 2015), which has been approximately constant over the last few megayears.

The total energy $E_{\mathrm{bol}}$ produced by the starburst is simply the time-integrated bolometric luminosity $L_{\mathrm{bol}}$, which depends ${ }^{14}$ on the bolometric magnitude $M_{\mathrm{bol}}$ :

$$
E_{\mathrm{bol}}=L_{\mathrm{bol}} \times \Delta t
$$

$$
=3 \times 10^{35-0.4 M_{\text {bol }}} \Delta t\left(\frac{\mathrm{SFR}}{1 M_{\odot} \mathrm{yr}^{-1}}\right) \mathrm{erg} \mathrm{s}^{-1} .
$$

\footnotetext{
${ }^{14}$ We follow IAU resolution B2 that defines the bolometric magnitude in absolute terms and eliminates the dependence on the variable magnitude of the Sun.
} 
According to Starburst99 (Figure 46 in Leitherer et al. 1999), the bolometric magnitude of a starburst at an age of $10^{7}-10^{8} \mathrm{yr}$ is $M_{\text {bol }} \sim-20.5$ for SFR $=1 M_{\odot} \mathrm{yr}^{-1}$. The total energy output of the starburst over the past $1 \mathrm{Myr}$ is thus $4.2 \times 10^{57} \mathrm{erg}$. The observed kinetic energy $\sim 3.9-4.5 \times 10^{54}$ erg in the outflow is a factor of $\sim 10^{3}$ lower, which places the coupling efficiency of the outflow kinetic energy to starburst energy at $\sim 0.1 \%$.

In terms of only kinetic energy, the fraction is higher. It is primarily supernovae and winds that supply kinetic energy to the ISM, which can be estimated from the energy deposition rate according to Leitherer et al. (1999) as given in Chisholm et al. (2017) and Murray et al. (2005):

$$
\dot{E}_{\mathrm{SN}}=3 \times 10^{41}\left(\frac{\mathrm{SFR}}{1 M_{\odot} \mathrm{yr}^{-1}}\right) \mathrm{erg} \mathrm{s}^{-1} .
$$

Each SN releases approximately $10^{51}$ erg in kinetic energy, with the progenitor releasing a similar amount of kinetic energy by winds during its lifetime (e.g., Leitherer et al. 1999). The approximate total kinetic energy released by $\mathrm{SNe}$ in the past 1 Myr is then $\sim 5.3 \times 10^{55} \mathrm{erg}$, compared to the $\sim 3.9-4.5 \times$ $10^{54}$ erg we observe in the outflow. Hence, the observed starburst is sufficient to kinetically power the measured molecular outflows with $\sim 8 \%$ efficiency.

The commonly adopted $50 \%$ relative contribution of wind feedback is a first-order estimate that is subject to environmental dependence and requires careful modeling to determine precisely (e.g., Leitherer et al. 1999). Furthermore, it should be noted that the observed outflow energy and its error are based on a fixed mass conversion factor that may vary. The uncertainty on the energy coupling efficiency is thus substantial, and it should be understood as an order-of-magnitude comparison.

The above calculation ignores the contribution of other energies, such as the turbulent energy within the molecular outflow and the kinetic energy of the neutral and ionized gases. Matsubayashi et al. (2009) derived a kinetic energy of the ionized wind in NGC 253 of $1.3 \times 10^{53} \mathrm{erg}$ or more than one order of magnitude lower than the molecular outflow kinetic energy. The molecular outflow is slower $\left(50-100 \mathrm{~km} \mathrm{~s}^{-1}\right.$ on the scales we observed here) than the ionized outflow (up to $\sim 400 \mathrm{~km} \mathrm{~s}^{-1}$; Matsubayashi et al. 2009) but also more massive. The ionized outflow thus has only a very small effect on the total kinetic energy and the coupling efficiency.

Deprojected outflow momenta integrated over the past $1.0 \mathrm{Myr}$ are $6.9 \times 10^{8} M_{\odot} \mathrm{km} \mathrm{s}^{-1}\left({ }_{-0.49}^{+0.50} \mathrm{dex}\right), 8.7 \times 10^{8} M_{\odot} \mathrm{km} \mathrm{s}^{-1}\left(_{-0.57}^{+0.57}\right.$ dex), and $1.2 \times 10^{8} M_{\odot} \mathrm{km} \mathrm{s}^{-1}\left({ }_{-0.59}^{+0.33} \mathrm{dex}\right)$ for $\mathrm{CO}(1-0),(2-1)$, and (3-2), respectively. Within $20^{\prime \prime}(340 \mathrm{pc}$ ) deprojected distance from the launching site, the outflow momenta integrate to $4.8 \times$ $10^{8} M_{\odot} \mathrm{km} \mathrm{s}^{-1}\left({ }_{-0.35}^{+0.48} \mathrm{dex}\right)$ in $\mathrm{CO}(1-0), 6.4 \times 10^{8} M_{\odot} \mathrm{km} \mathrm{s}^{-1}$ $\left({ }_{-0.34}^{+0.49}\right.$ dex $)$ in $\mathrm{CO}(2-1)$, and $\left.8.0 \times 10^{7} M_{\odot} \mathrm{km} \mathrm{s}^{-1}{ }_{-0.39}^{+0.50} \mathrm{dex}\right)$ in $\mathrm{CO}(3-2)$.

The momentum released initially by $\mathrm{SNe}$ is given in Murray et al. (2005):

$$
\begin{aligned}
& \dot{P}_{\mathrm{SN}}=2 \times 10^{33}\left(\frac{\mathrm{SFR}}{1 M_{\odot} \mathrm{yr}^{-1}}\right) \mathrm{g} \mathrm{cm} \mathrm{s}^{-2} \\
& =317\left(\frac{\mathrm{SFR}}{1 M_{\odot} \mathrm{yr}^{-1}}\right) M_{\odot} \mathrm{yr}^{-1} \mathrm{~km} \mathrm{~s}^{-1} .
\end{aligned}
$$

In $1 \mathrm{Myr}$, a constant $\mathrm{SFR}$ of $2.8 \mathrm{M}_{\odot} \mathrm{yr}^{-1}$ yields $8.9 \times$ $10^{8} M_{\odot} \mathrm{km} \mathrm{s}^{-1}$. Assuming a contribution by stellar winds of the same order (Leitherer et al. 1999), the total momentum is $1.8 \times 10^{9} M_{\odot} \mathrm{km} \mathrm{s}^{-1}$, or roughly twice the observed outflow momentum. SNe, however, gain significant amounts ${ }^{15}$ of momentum by sweeping up surrounding material. From simulations, the total momentum supplied to the ISM is expected to be $2.8 \times 10^{5} M_{\odot} \mathrm{km} \mathrm{s}^{-1}$ per SNe (Kim \& Ostriker 2015 and references therein). For a constant SFR of $2.8 M_{\odot} \mathrm{yr}^{-1}$ over $1 \mathrm{Myr}$, this amounts to $1.0 \times 10^{10} M_{\odot} \mathrm{km} \mathrm{s}^{-1}$ or $2.0 \times$ $10^{10} M_{\odot} \mathrm{km} \mathrm{s}^{-1}$ when adopting $50 \%$ contribution by stellar winds, which is about four times the observed momentum. The efficiency of transferring feedback momentum to outflow momentum is thus in the range of $27 \%-49 \%$, considering the initially available momentum, or a $2.5 \%-4 \%$ efficiency for total to outflow momentum transfer.

These outflow momenta are much higher than the momentum currently produced by young $(<10 \mathrm{Myr})$ super star clusters in the starburst. Leroy et al. (2018) list 14 candidate clusters that together produce $1.5 \times 10^{7} M_{\odot} \mathrm{km} \mathrm{s}^{-1}$ measured from gas kinematics, a factor 10-100 lower than the observed outflow momentum. The currently forming (super) star cluster thus could not have launched the outflow but the feedback of another population of stars is needed to explain the observed outflows. This is indicative of the time delay of SF feedback.

Energy and momentum curves in Figure 9 differ only by a factor $v$ but follow a similar evolution. This implies that the median velocity at a given distance must be roughly constant along the outflow. As the curves as a function of distance are roughly constant within $50 \mathrm{pc}<s<300 \mathrm{pc}$, especially for kinetic energy, the outflow mass at a given distance must also be approximately constant along the outflow. The decline in energy and momentum below $50 \mathrm{pc}$ is caused by a decrease in outflow mass, again because both curves follow a similar trend. This is at least partially related to the difficulty of separating the outflow from the disk, where the former emerges from the latter. The decrease could also be interpreted physically as the outflow sweeping up mass while emerging from the disk. An estimation of the relative importance of these effects requires high-resolution modeling of the outflow that are not possible yet because we do not know the detailed outflow geometry. The drop beyond $\sim 300 \mathrm{pc}(\sim 200 \mathrm{pc}$ in $\mathrm{CO}(3-2))$ is partially related to reaching the edge of the field of view. Discerning this effect from an actual decrease is not possible with our data as we do not know the inclination at every location in the outflow. The edge of the field of view thus corresponds to a range of deprojected distances from the disk, which gradually depresses the curve rather than showing a sudden drop. A physical reason for the decrease could be the destruction of the molecular gas, e.g., photodissociation by the intense starburst radiation or ionization.

The kinetic energy and momentum evolution in Figure 9 thus suggest both energy and momentum conservation along the outflow from $\sim 50 \mathrm{pc}$ to $\sim 300 \mathrm{pc}$, as well as approximately constant molecular gas mass.

When additionally assuming no acceleration of the outflow after launch, it becomes possible to study the time evolution. The corresponding plots (Figure 8 top and 9 top) all show a peak within the past $0.5 \mathrm{Myr}$ and steady decrease toward earlier gas ejection times. Corresponding to the decline toward zero

\footnotetext{
15 Assuming a Salpeter-like IMF $\left(\alpha=2.35\right.$, mass range $0.1-100 \mathrm{M}_{\odot}$, $Z=0.008$ ). The usual uncertainties related to the shape, upper mass cutoff, and influence of binary stars apply.
} 
distance, the decrease toward zero ejection time is most likely a methodological complication. From the peak at $t_{\text {eject }}=0$. 2-0.3 Myr, kinetic energy and momentum in the outflow drop by a factor of 10 within $\sim 2 \mathrm{Myr}$. This decline would be physically plausible if the starburst in NGC 253 is very young and takes into account a time delay between the start of star formation, feedback, and efficient outflow driving (superbubble breakout). For the observed age of the starburst of 20-30 Myr (Rieke et al. 1980; Engelbracht et al. 1998) this scenario is implausible. Time delays of $>20 \mathrm{Myr}$ are longer than the lifetime of the most massive stars. A younger generation of massive stars at an age of $\sim 6 \mathrm{Myr}$ (Kornei \& McCrady 2009) may, however, drive the currently visible molecular outflows. If this were to be true, a time delay between star formation and outflow launching of $\sim 4 \mathrm{Myr}$ is implied. Outflow launching in this context means the time after which the outflow reaches a mass loading $\eta>1$. The time delay is $2 \mathrm{Myr}$ until the outflow carries more energy (momentum) than the feedback kinetic energy (momentum) of a single high-mass star. Note that these rough estimates depend on the assumption of no acceleration (neither positive nor negative) of the outflow after being launched from the disk, which might be a close enough approximation on these scales of a few hundred parsecs. The very young $(\lesssim 1 \mathrm{Myr})$ and still deeply embedded super star cluster discussed recently by Ando et al. (2017) and Leroy et al. (2018) are most likely too young to have affected the observed molecular outflow.

\section{Summary and Conclusions}

We present $\mathrm{CO}(3-2)$ observations taken with ALMA that offer an unprecedented resolution of $\sim 0$ " $15(\sim 2 \mathrm{pc})$ in the starbursting center of NGC 253. The new high-resolution data show structures consistent with previous lower resolution observations in other $\mathrm{CO}$ lines, revealing the complexity of the molecular ISM in a starburst on scales of a few parsecs.

We use archival $\mathrm{CO}(1-0), \mathrm{CO}(2-1)$, and the new $\mathrm{CO}(3-2)$ ALMA observations to perform a ppV decomposition of the emission into different structures. The bulk of the emission is associated with a rotating disk with streaming motions due to the bar. The rest of the emission is incompatible with a simple kinematic model of a disk plus a bar. This "nondisk" component is further decomposed into an outflow, an expanding superbubble (part of which may be associated with outflowing gas), and a potential second kinematic component within the disk.

We find $\mathrm{CO}$ line luminosities of the disk component of $2.8 \times 10^{8} \mathrm{~K} \mathrm{~km} \mathrm{~s}^{-1} \mathrm{pc}^{2}, 2.3 \times 10^{8} \mathrm{~K} \mathrm{~km} \mathrm{~s}^{-1} \mathrm{pc}^{2}$, and $1.8 \times$ $10^{8} \mathrm{~K} \mathrm{~km} \mathrm{~s}^{-1} \mathrm{pc}^{2}$ for $\mathrm{CO}(1-0)$, (2-1), and (3-2), respectively. The fractional luminosity of the nondisk component is small, amounting to $\sim 7 \%-16 \%$ of the total. A significant amount of the outflow emission we identify is faint and diffuse, while part of the emission is in discrete, higher surface brightness structures (e.g., the SW streamer).

Assuming a starburst conversion factor, we estimate the molecular gas mass from the three $\mathrm{CO}$ transitions. Masses match within $10 \%$ for the disk component and within $50 \%$ for the nondisk component. The total gas mass in the center of NGC 253 is $\sim 3.6 \times 10^{8} M_{\odot}$, with $\sim 0.5 \times 10^{8} M_{\odot}$ in the nondisk component.

We further estimate the deprojected molecular mass outflow rate, kinetic energy, and momentum in the starburst of NGC 253. The observed gas distribution can be interpreted to have formed in two ways: (1) by a constant starting mass outflow rate over the lifetime of the starburst and (2) through continuous gas ejection without acceleration of the gas after ejection. In the first interpretation, the molecular mass outflow rate averaged over a deprojected distance of $340 \mathrm{pc}\left(20^{\prime \prime}\right)$ from the launching site is $29-39 M_{\odot} \mathrm{yr}^{-1}$. Typical uncertainties are 0.4 dex. The majority of this outflow rate is contributed by massive localized features such as the SW/SE streamers, with a significant contribution by diffuse molecular gas. The massloading factor $\eta=\dot{M}_{\mathrm{SFR}} / \dot{M}_{\text {out }} \sim 14-20$ is relatively high. Due to the limited field of view of our observations, this $\eta$ applies to gas ejected as far away as $340 \mathrm{pc}$ : the fraction of mass that makes it to the far regions of the halo or escapes is not known. The kinetic energy of the molecular outflow within $340 \mathrm{pc}$ from the launching site is $2.5-3.1 \times 10^{54} \mathrm{erg}$ with a $\sim 0.8$ dex error. The coupling efficiency of kinetic energy in the outflow to the total energy released by the starburst is $\sim 0.1 \%$ while the coupling to only the kinetic energy is higher at $\sim 8 \%$. Including other phases of the outflow would increase this efficiency. The kinetic energy of the ionized outflow is negligible relative to the molecular outflow. The outflow momenta within the same distance are 4.8-6.4 $\times 10^{8} M_{\odot} \mathrm{km} \mathrm{s}^{-1}$ (error $\sim 0.5 \mathrm{dex}$ ) which is $\sim 2.5 \%-4 \%$ of the momentum supplied by $\mathrm{SNe}$ and winds. These best estimates for the physical properties of the outflow are derived from the $\mathrm{CO}(1-0)$ and $(2-1)$ observations. The very high resolution of the $\mathrm{CO}(3-2)$ data is necessary to identify the outflow features that connect to the central regions.

When interpreting the outflow as a structure of constant velocity along the outflow, the time evolution can be reconstructed. We derive the outflow rate, kinetic energy, and momentum within the approximate dynamical timescale of $1 \mathrm{Myr}$ and find lower values compared to the previous interpretation. The difference is systematic at the $\sim 30 \%-40 \%$ level. The outflow rate is $14-20 M_{\odot} \mathrm{yr}^{-1}(0.3 \mathrm{dex})$, kinetic energy $2.5-3.1 \times 10^{54} \mathrm{erg}$ (0.8 dex), and momentum 4.8-6.4 × $10^{8} M_{\odot} \mathrm{km} \mathrm{s}^{-1}(0.5 \mathrm{dex})$.

For all measurements given above, we assume a fixed starburst mass conversion factor of $X_{\mathrm{CO}}=0.5 \times 10^{20}\left(\mathrm{~K} \mathrm{~km} \mathrm{~s}^{-1}\right)^{-1}$. The quoted uncertainties are primarily systematic, due to the unknown geometry of the outflow and its launching sites. A further uncertainty of $30 \%-40 \%(\sim 0.1$ dex $)$ comes from the assumptions regarding the outflowing material (constant starting mass over the lifetime of the starburst versus continuous gas ejection without acceleration). These limitations need to be addressed in the future. In principle, ALMA can also provide the very high resolution and sensitivity needed to enable this detailed view of a starburst on larger scales than probed in this study.

The authors thank Jan-Torge Schindler and Roberto Decarli for insightful discussion and advice. This paper makes use of the following ALMA data: ADS/JAO.ALMA \#2011.1.00172.S, \#2012.1.00108.S, and \#2015.1.00274.S. ALMA is a partnership of ESO (representing its member states), NSF (USA) and NINS (Japan), together with NRC (Canada), NSC and ASIAA (Taiwan), and KASI (Republic of Korea), in cooperation with the Republic of Chile. The Joint ALMA Observatory is operated by ESO, AUI/NRAO, and NAOJ. The National Radio Astronomy Observatory is a facility of the National Science Foundation operated under cooperative agreement by Associated Universities, Inc. Part of the work presented in this paper was carried out at the Finnish Center for Astronomy with ESO (FINCA).

The work of A.K.L. is partially supported by NASA ADAP grants NNX16AF48G and NNX17AF39G and the National Science Foundation under grants No. 1615105, 1615109, and 1653300 . 
Software: CASA (McMullin et al. 2007), astropy (Astropy Collaboration et al. 2013, 2018), APLpy (Robitaille \& Bressert 2012).

\section{Appendix A \\ Kinematic Model of the Central Molecular Gas}

We derive a model for the velocity of the disk component from the $\mathrm{CO}(1-0)$ observations using the kinematic fitting tool diskfit (Spekkens \& Sellwood 2007; Sellwood \& Sánchez 2010; Sellwood \& Spekkens 2015). As mentioned in Section 3.1, these models benefit from the large area, which is why we base them on the $\mathrm{CO}(1-0)$ observations. A $20 \sigma$ threshold ensures that the model is fitted to the bright disk, excluding any fainter outflows.

In diskfit, we fit using the velocity field fitter. The names of the set options in diskfit are given in parentheses in the following. The model is fitted to all pixels within an ellipse of
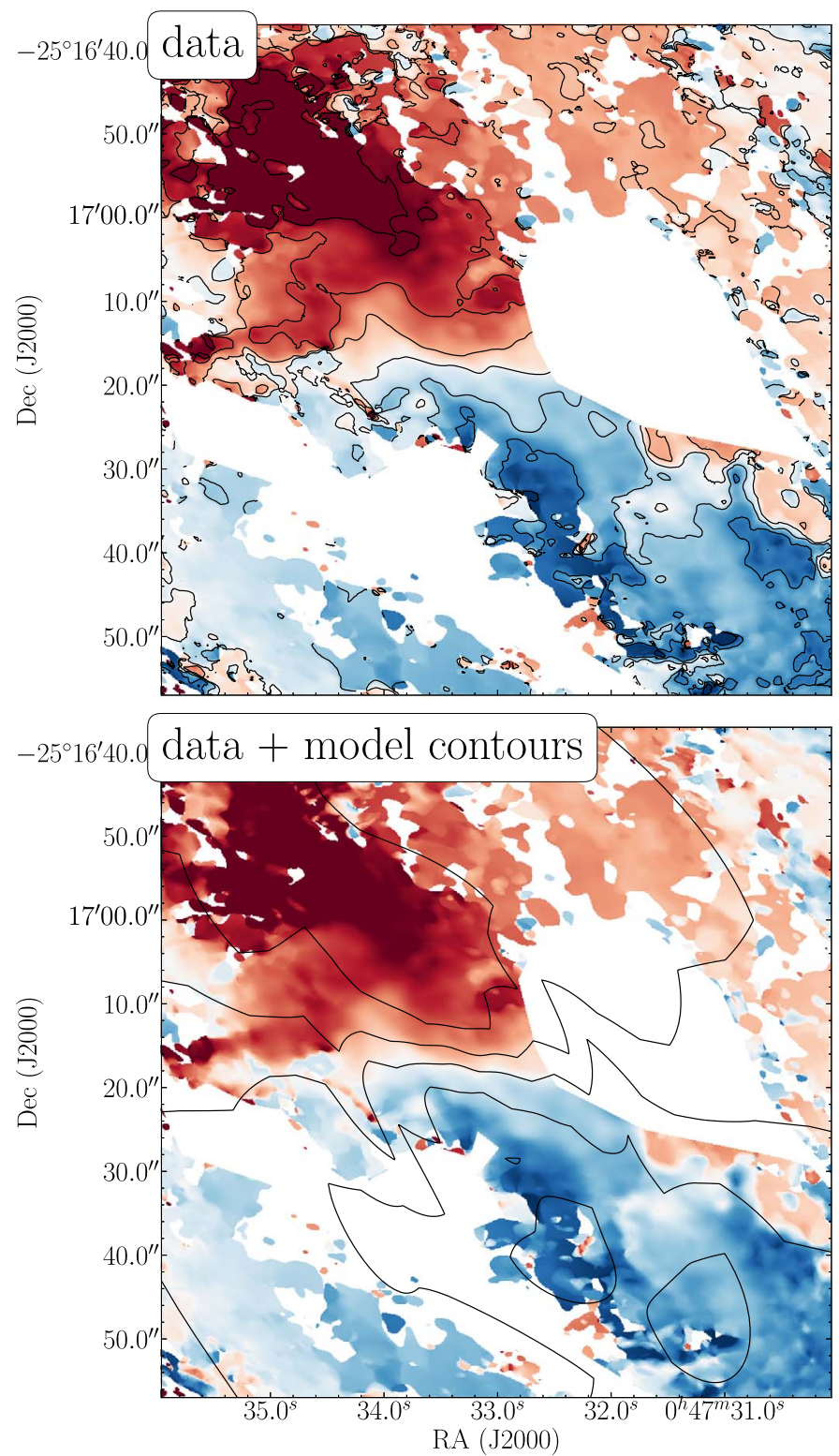

$75^{\prime \prime}$ major axis length (regrad), $\mathrm{PA}=53^{\circ}$ (regpa), and ellipticity $\epsilon=0.66$ (regeps). Outside this range, we use a sampling factor of 2 pixels (istepout). During the fit, the center is held fixed while we fit for the disk position angle and ellipticity with initial guesses of $\mathrm{PA}=53^{\circ}$ and $\epsilon=0.66$ based on by-eye inspection (lines 9 and 10 of the parameter file). We allow the model to fit for nonaxisymmetric flows with a $\mathrm{PA}=78^{\circ}$ initial guess and order $m=2$ (line 12), which means diskfit will fit for rotation plus a bisymmetric model with $m=2$ perturbations to the potential (bar). As the $\mathrm{CO}(1-0)$ data cover the kinematic center, we set the inner interpolation toggle to true, which assumes the velocity raises linearly within the innermost fitted ring. We do not fit for radial flows (radial flows toggle) because it allows too many degrees of freedom and produces bad models, as warned in the diskfit manual. We further fit for the systemic velocity and exclude warps from the
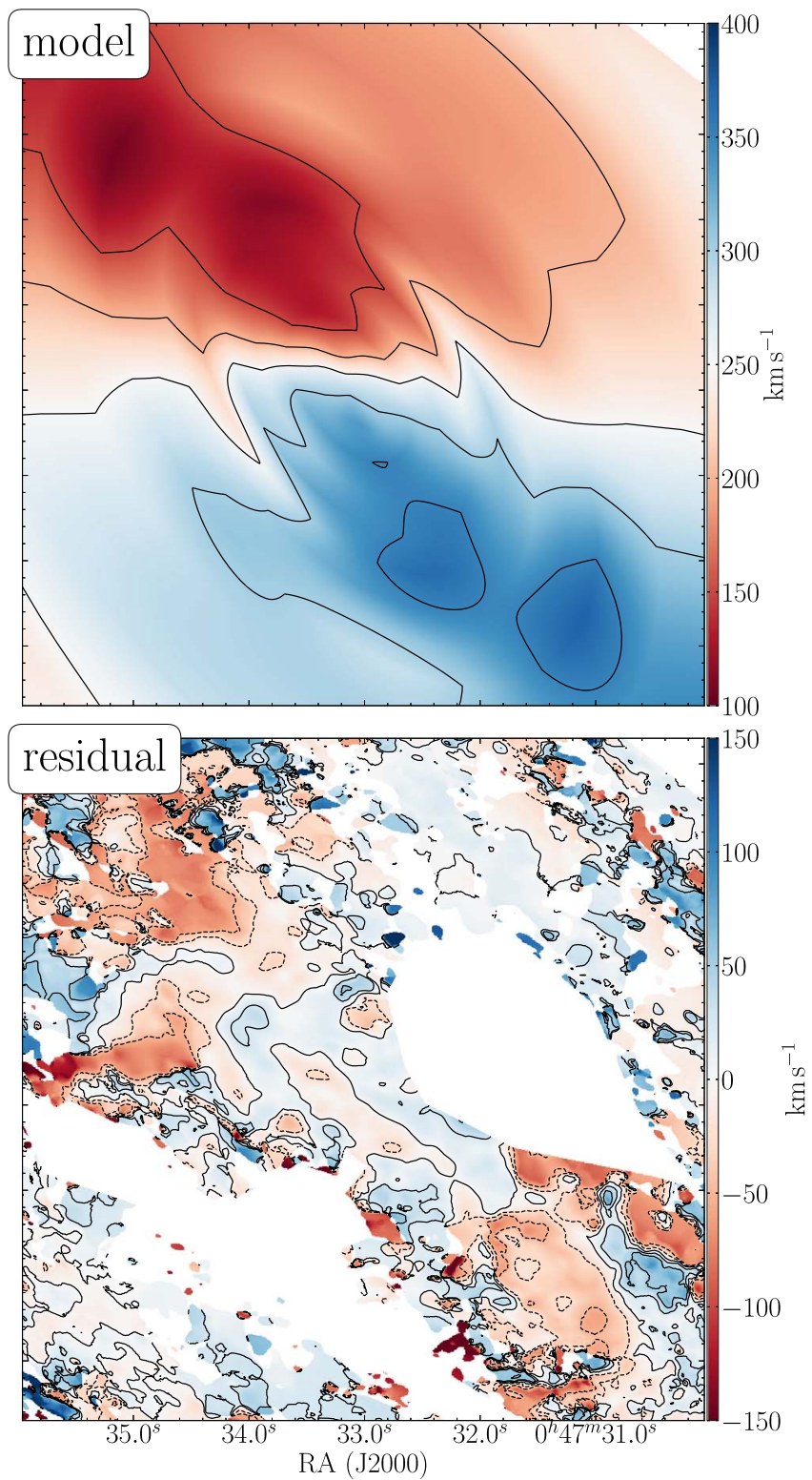

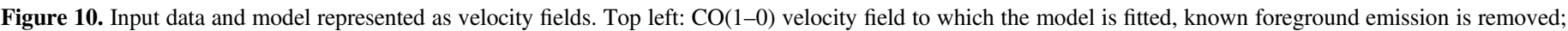

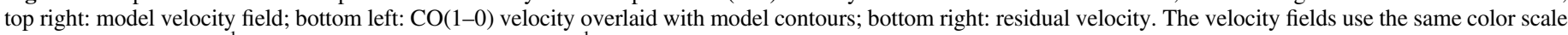

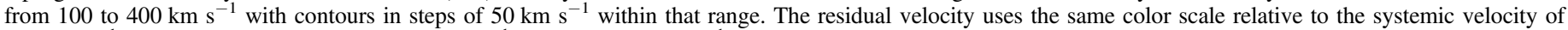
$250 \mathrm{~km} \mathrm{~s}^{-1}$ with contours from -50 to $+50 \mathrm{~km} \mathrm{~s}^{-1}$ in steps of $25 \mathrm{~km} \mathrm{~s}^{-1}$. 
model. A model with these parameters is fitted in rings at radii

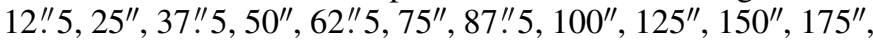
$200^{\prime \prime}, 225^{\prime \prime}, 250^{\prime \prime}, 275^{\prime \prime}$, and $300^{\prime \prime}$.

The residuals show a slight mismatch in velocity of $\sim 20-30 \mathrm{~km} \mathrm{~s}^{-1}$ along the direction of the bar. This is likely due to the bar being underestimated because the $\mathrm{CO}(1-0)$ image covers only the inner half of the total extent of the bar. The mismatch gets larger when fitting a model to the smaller images of $\mathrm{CO}(2-1)$ and (3-2), which confirms that it is caused by the lack of observed area. We fit this mismatch in the velocity field with an additional 2D Gaussian component and add it to the velocity field of the diskfit model to obtain a better model. Note that this additional component is not physically motivated or meaningful but purely aims to counteract the effect of limited observation area.

Figure 10 shows the velocity field of the model in comparison to the input $\mathrm{CO}(1-0)$ velocity field. The model typically fits the observed velocity field better than $\pm 25 \mathrm{~km} \mathrm{~s}^{-1}$; larger deviations occur mostly over small areas of order one beam size. The model thus successfully reproduces the large-scale velocity field.

\section{Appendix B}

\section{Velocity Width of the Disk Mask}

The definition of disk mask is crucial for this analysis as it determines whether a molecular cloud is considered kinematically consistent with the disk or if it is potentially outflowing. The position of the disk mask in $\mathrm{ppV}$ space is set by the disk model, but the width (velocity range $\Delta v$ ) of the mask is a free parameter. From Figure 12, it is obvious that $\Delta v$ depends on the distance from the major axis, which is most simply accounted for by a parameterization of the form $\Delta v=a \exp \left(-\left(\frac{x}{b}\right)^{2}\right)+c$. Finding the best-fit values for $a, b$, and $c$ is difficult to do mathematically as the fit would need to be on the disk component that we want to determine from the mask first. We therefore select values that visually fit the $\mathrm{pV}$ diagrams as best as possible. They are given in Equation (1).

Figure 11 shows five alternative masks that vary only in width by $10 \%$ from the best-fit mask. It is apparent that even slight changes of $10 \%$ deteriorate the fit between mask and disk emission. Narrower masks obviously do not cover all disk emission, whereas the wider masks include spike features that are kinematically inconsistent with disk rotation.

The effect of a ${ }_{-10}^{+10} \%\left({ }_{-0.04}^{+0.04} \mathrm{dex}\right)$ variation in the mask velocity width results in a ${ }_{-0.01}^{+0.01}$ dex change in the integrated disk luminosity for $\mathrm{CO}(1-0), \mathrm{CO}(2-1)$, and $\mathrm{CO}(3-2)$. The nondisk components are less bright than the disk and thus shows a higher relative variation when changing the mask width: luminosities vary by ${ }_{+0.08}^{-0.07} \mathrm{dex}\left(\begin{array}{l}-0.06 \\ +0.07 \\ \mathrm{dex},\end{array}+0.12 \mathrm{dex}\right)$ for $\mathrm{CO}(1-0)(\mathrm{CO}(2-1), \mathrm{CO}(3-2))$. Note the inverse scaling

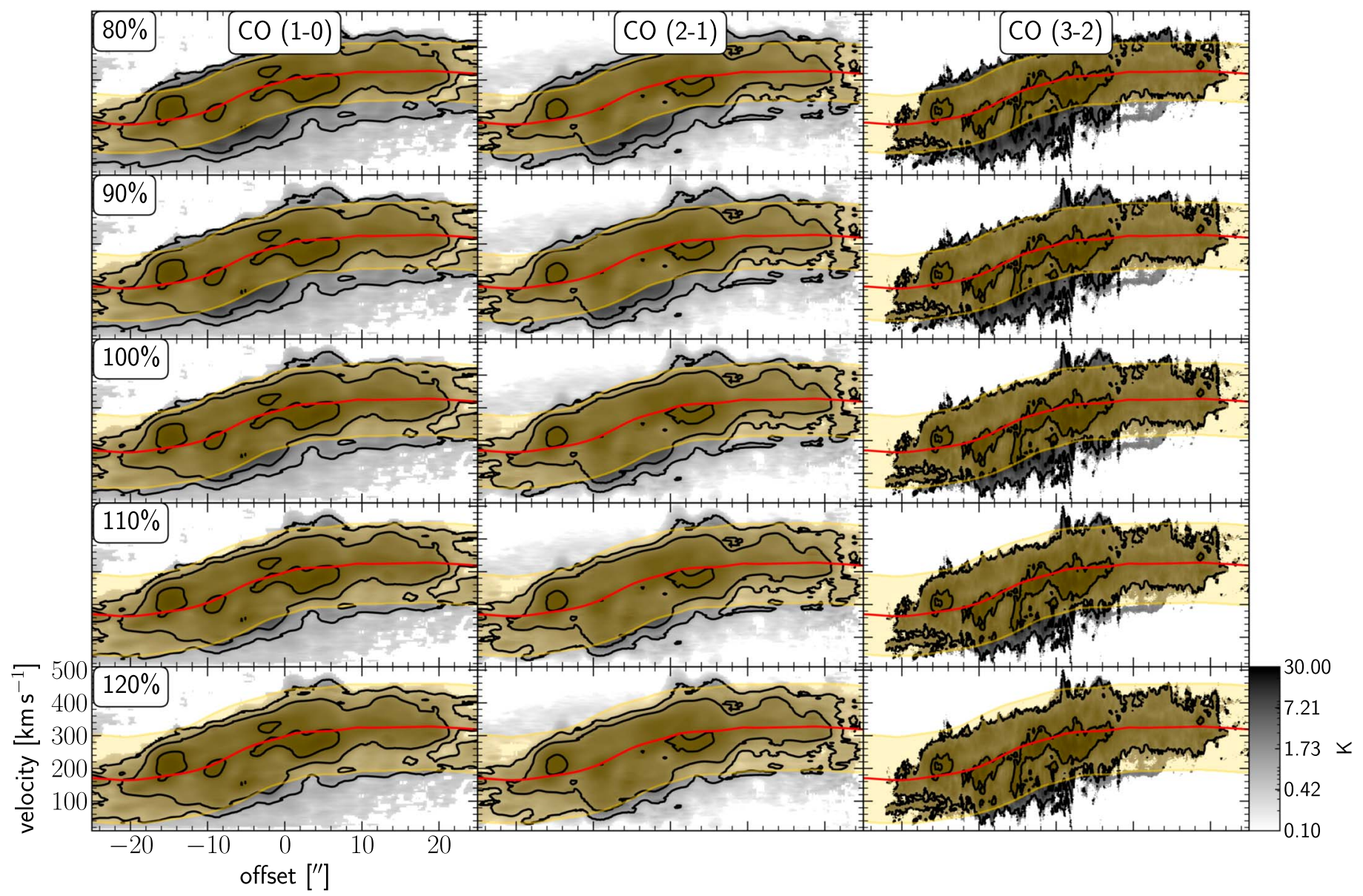

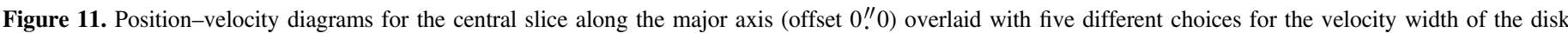

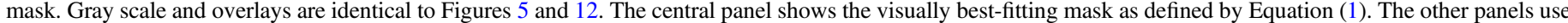

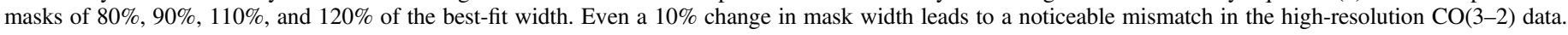


between disk and nondisk, due to the balance shifting between the two components for a constant total luminosity. To first order, the same percentage changes apply to the other quantities mass, outflow rate, energy, and momentum.

\section{Appendix C \\ Disk/Nondisk Separation: pV Diagrams}

Figure 12 shows all $\mathrm{pV}$ diagrams for the slices defined in Figure 4. For the discussion on these diagrams, see Section 3.

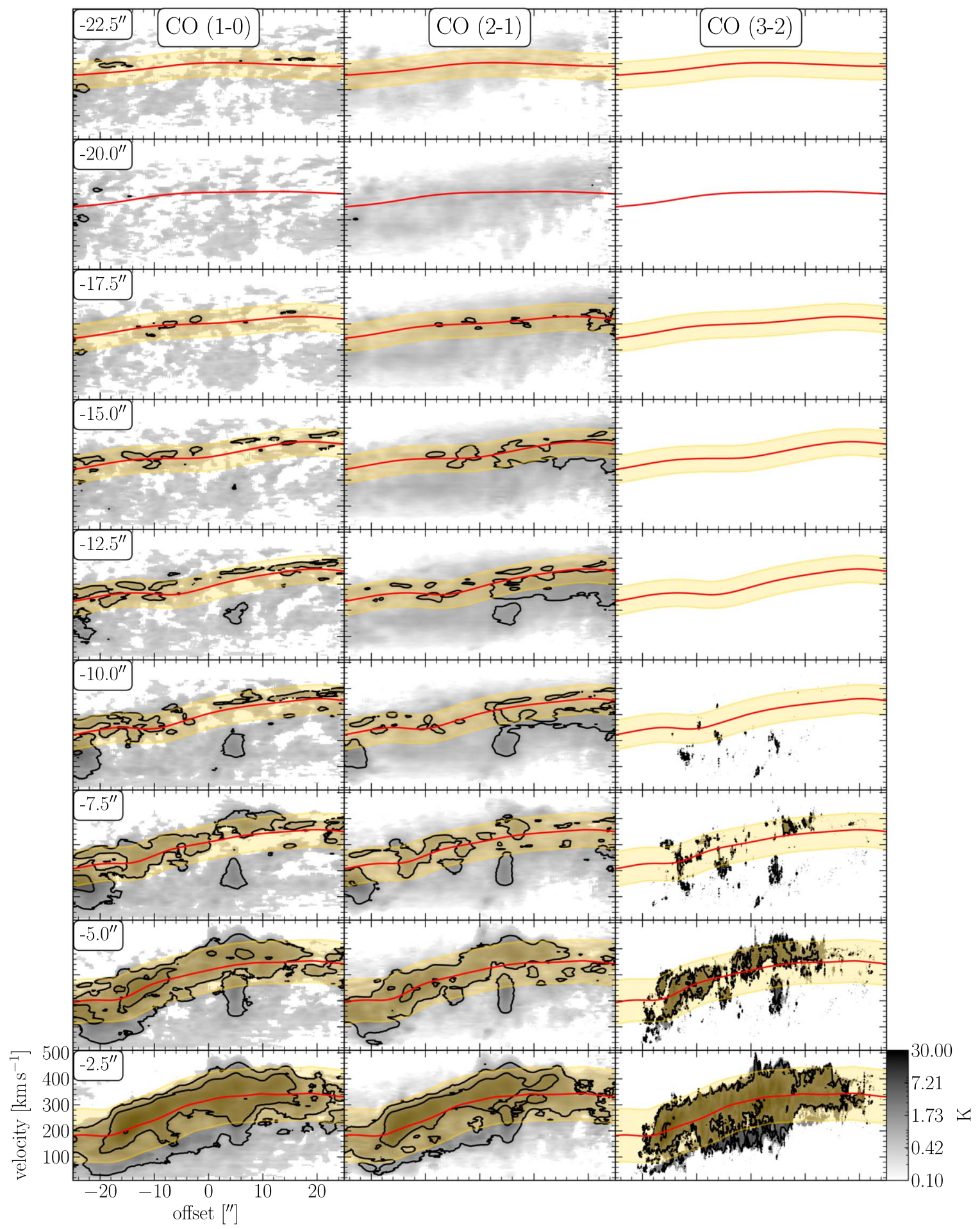

Figure 12. Position-velocity diagrams for all slices defined in Section 3 and Figure 4 for $\operatorname{CO}(1-0), \operatorname{CO}(2-1)$, and $\operatorname{CO}(3-2)$ from the left to right columns. A description of the overlays is given in Figure 5. 


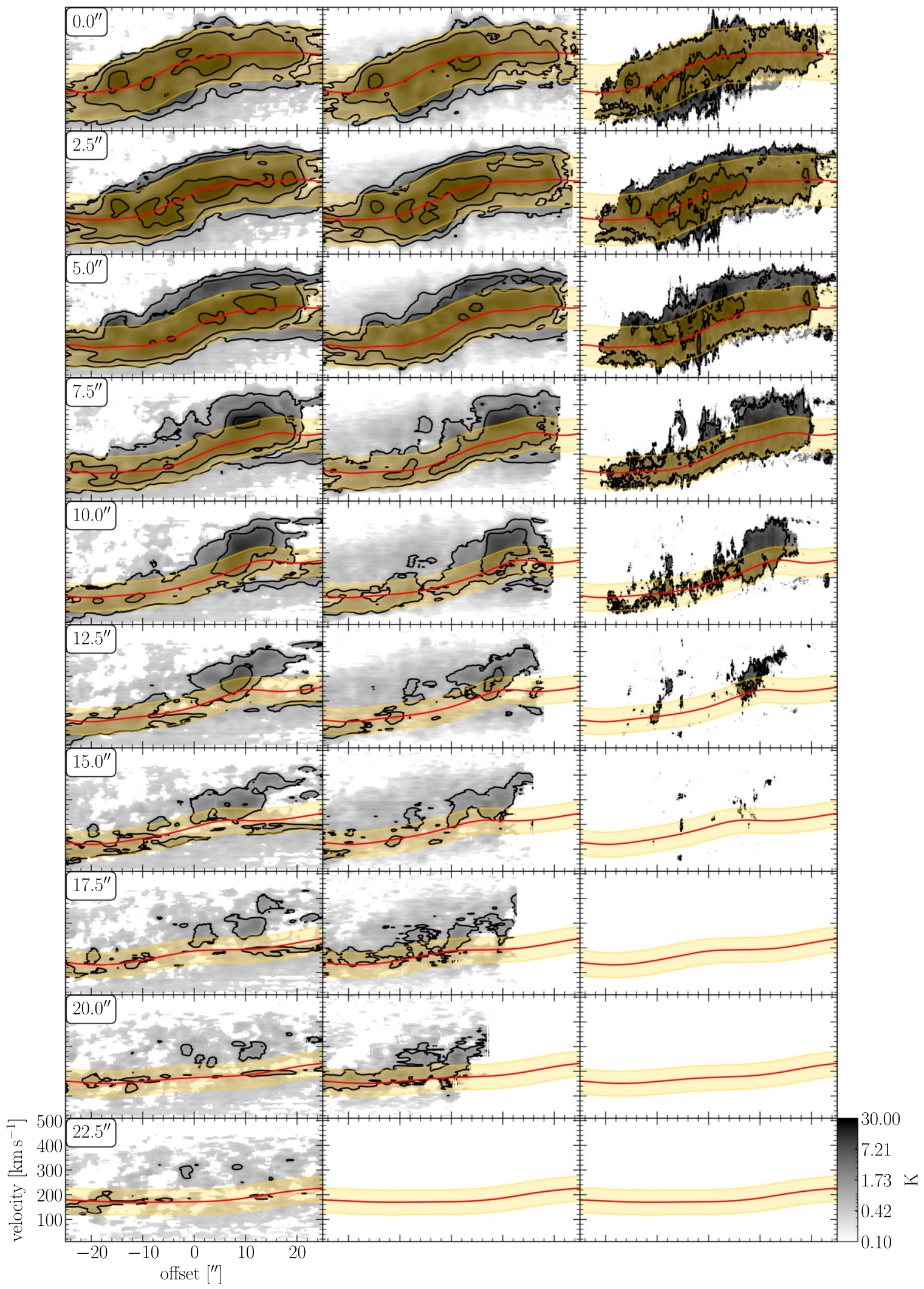

Figure 12. (Continued.)

\section{ORCID iDs}

Nico Krieger (ㄴ) https://orcid.org/0000-0003-1104-2014

Alberto D. Bolatto (i) https://orcid.org/0000-0002-5480-5686
Fabian Walter (1) https://orcid.org/0000-0003-4793-7880 Adam K. Leroy (1) https://orcid.org/0000-0002-2545-1700 Laura K. Zschaechner (i) https://orcid.org/0000-0002-9919-8672 
David S. Meier (1) https://orcid.org/0000-0001-9436-9471

Jürgen Ott (i) https://orcid.org/0000-0001-8224-1956

Axel Weiss (10 https://orcid.org/0000-0003-4678-3939

Elisabeth A. C. Mills (i) https://orcid.org/0000-0001-8782-1992

Rebecca C. Levy (1) https://orcid.org/0000-0003-2508-2586

Sylvain Veilleux (i) https://orcid.org/0000-0002-3158-6820

\section{References}

Ando, R., Nakanishi, K., Kohno, K., et al. 2017, ApJ, 849, 81

Astropy Collaboration, Price-Whelan, A. M., Sipőcz, B. M., et al. 2018, AJ, 156,123

Astropy Collaboration, Robitaille, T. P., Tollerud, E. J., et al. 2013, A\&A, 558, A33

Bendo, G. J., Beswick, R. J., D’Cruze, M. J., et al. 2015, MNRAS, 450, L80

Bolatto, A. D., Warren, S. R., Leroy, A. K., et al. 2013, Natur, 499, 450

Chisholm, J., Tremonti, C. A., Leitherer, C., \& Chen, Y. 2017, MNRAS, 469,4831

Christensen, C. R., Davé, R., Brooks, A., Quinn, T., \& Shen, S. 2018, ApJ, 867,142

Dekel, A., \& Silk, J. 1986, ApJ, 303, 39

Engelbracht, C. W., Kundurthy, P., Gordon, K. D., et al. 2006, ApJL, 642, L127

Engelbracht, C. W., Rieke, M. J., Rieke, G. H., Kelly, D. M., \& Achtermann, J. M. 1998, ApJ, 505, 639

Heckman, T. M., Lehnert, M. D., Strickland, D. K., \& Armus, L. 2000, ApJS, 129,493

Henshaw, J. D., Longmore, S. N., Kruijssen, J. M. D., et al. 2016, MNRAS, 457, 2675

Hoopes, C. G., Heckman, T. M., Strickland, D. K., et al. 2005, ApJL, 619, L99

Hopkins, P. F., Quataert, E., \& Murray, N. 2012, MNRAS, 421, 3522

Kim, C.-G., \& Ostriker, E. C. 2015, ApJ, 802, 99

Kim, C.-G., \& Ostriker, E. C. 2018, ApJ, 853, 173

Kornei, K. A., \& McCrady, N. 2009, ApJ, 697, 1180

Krumholz, M. R., \& Kruijssen, J. M. D. 2015, MNRAS, 453, 739

Krumholz, M. R., Kruijssen, J. M. D., \& Crocker, R. M. 2017, MNRAS, 466, 1213

Leitherer, C., Schaerer, D., Goldader, J. D., et al. 1999, ApJS, 123, 3

Leroy, A. K., Bolatto, A. D., Ostriker, E. C., et al. 2015a, ApJ, 801, 25

Leroy, A. K., Bolatto, A. D., Ostriker, E. C., et al. 2018, ApJ, 869, 126

Leroy, A. K., Walter, F., Martini, P., et al. 2015b, ApJ, 814, 83

Lucero, D. M., Carignan, C., Elson, E. C., et al. 2015, MNRAS, 450, 3935

Ma, C., de Grijs, R., \& Ho, L. C. 2018, ApJ, 857, 116

Mangum, J. G., Ginsburg, A. G., Henkel, C., et al. 2019, ApJ, 871, 170

Matsubayashi, K., Sugai, H., Hattori, T., et al. 2009, ApJ, 701, 1636
Mauersberger, R., Henkel, C., Wielebinski, R., Wiklind, T., \& Reuter, H. P. 1996, A\&A, 305, 421

McMullin, J. P., Waters, B., Schiebel, D., Young, W., \& Golap, K. 2007, adass XVI, 376, 127

Meier, D. S., Walter, F., Bolatto, A. D., et al. 2015, ApJ, 801, 63

Müller-Sánchez, F., González-Martín, O., Fernández-Ontiveros, J. A., Acosta-Pulido, J. A., \& Prieto, M. A. 2010, ApJ, 716, 1166

Murray, N., Quataert, E., \& Thompson, T. A. 2005, ApJ, 618, 569

Oppenheimer, B. D., \& Davé, R. 2006, MNRAS, 373, 1265

Oppenheimer, B. D., Davé, R., Kereš, D., et al. 2010, MNRAS, 406, 2325

Ott, J., Weiß, A., Henkel, C., \& Walter, F. 2005, ApJ, 629, 767

Paglione, T. A. D., Yam, O., Tosaki, T., \& Jackson, J. M. 2004, ApJ, 611, 835

Pereira-Santaella, M., Colina, L., García-Burillo, S., et al. 2016, A\&A, 594, A81

Pérez-Beaupuits, J. P., Güsten, R., Harris, A., et al. 2018, ApJ, 860, 23

Rekola, R., Richer, M. G., McCall, M. L., et al. 2005, MNRAS, 361, 330

Rieke, G. H., Lebofsky, M. J., Thompson, R. I., Low, F. J., \& Tokunaga, A. T. 1980, ApJ, 238, 24

Robitaille, T., \& Bressert, E. 2012, APLpy: Astronomical Plotting Library in Python, Astrophysics Source Code Library, ascl:1208.017

Roussel, H., Wilson, C. D., Vigroux, L., et al. 2010, A\&A, 518, L66

Sakamoto, K., Ho, P. T. P., Iono, D., et al. 2006, ApJ, 636, 685

Sakamoto, K., Mao, R.-Q., Matsushita, S., et al. 2011, ApJ, 735, 19

Salak, D., Nakai, N., Hatakeyama, T., \& Miyamoto, Y. 2016, ApJ, 823, 68

Salak, D., Tomiyasu, Y., Nakai, N., et al. 2018, ApJ, 856, 97

Sellwood, J. A., \& Sánchez, R. Z. 2010, MNRAS, 404, 1733

Sellwood, J. A., \& Spekkens, K. 2015, arXiv:1509.07120

Shapiro, P. R., \& Field, G. B. 1976, ApJ, 205, 762

Sharp, R. G., \& Bland-Hawthorn, J. 2010, ApJ, 711, 818

Sormani, M. C., Sobacchi, E., Fragkoudi, F., et al. 2018, MNRAS, 481, 2

Spekkens, K., \& Sellwood, J. A. 2007, ApJ, 664, 204

Strickland, D. K., \& Heckman, T. M. 2007, ApJ, 658, 258

Strickland, D. K., Heckman, T. M., Weaver, K. A., \& Dahlem, M. 2000, AJ, 120,2965

Strickland, D. K., Heckman, T. M., Weaver, K. A., Hoopes, C. G., \& Dahlem, M. 2002, ApJ, 568, 689

Sturm, E., González-Alfonso, E., Veilleux, S., et al. 2011, ApJL, 733, L16

Tollet, É., Cattaneo, A., Macciò, A. V., Dutton, A. A., \& Kang, X. 2019, MNRAS, 485, 2511

Turner, B. E. 1985, ApJ, 299, 312

Veilleux, S., Rupke, D. S. N., \& Swaters, R. 2009, ApJL, 700, L149

Walter, F., Bolatto, A. D., Leroy, A. K., et al. 2017, ApJ, 835, 265

Walter, F., Weiß, A., \& Scoville, N. 2002, ApJL, 580, L21

Westmoquette, M. S., Smith, L. J., Gallagher, J. S. I., et al. 2009, ApJ, 696, 192

Westmoquette, M. S., Smith, L. J., \& Gallagher, J. S., III 2011, MNRAS, 414, 3719

Zschaechner, L. K., Bolatto, A. D., Walter, F., et al. 2018, ApJ, 867, 111 